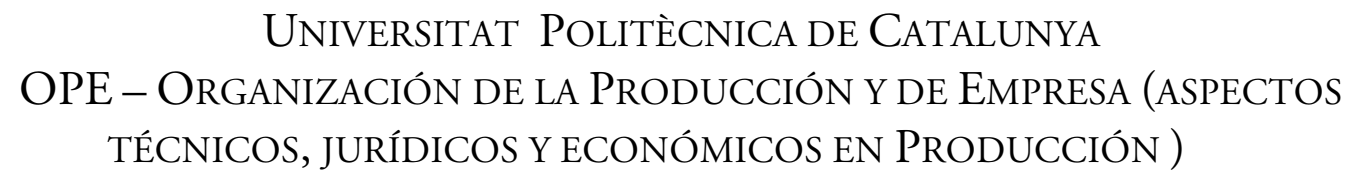

Consideration of human resources in the Mixed-model Sequencing Problem with Work Overload Minimization: Legal provisions and productivity improvement Joaquín Bautista, Rocío Alfaro-Pozo, Cristina Batalla-García, (ETSEIB-UPC)

OPE-WP.2015/08 (20151029)

(Postprint ESWA Volume 42, Issue 22, 1 December 2015, Pages 8896-8910) http://www.sciencedirect.com/science/article/pii/S0957417415005047

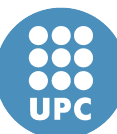




\title{
Consideration of human resources in the Mixed-model Sequencing Problem with Work Overload Minimization: Legal provisions and productivity improvement
}

\author{
Joaquín Bautista ${ }^{1}$, Rocío Alfaro-Pozo ${ }^{1}$, Cristina Batalla-García ${ }^{1}$ \\ ${ }^{1}$ Universitat Politècnica de Catalunya - BarcelonaTech
}

\begin{abstract}
Beginning with a variation of the sequencing problem in a mixed-products line (MMSP-W: Mixed-Model Sequencing Problem with Workload Minimization), we propose two new models that incorporate a set of working conditions in regard with human resources of workstations on the line. These conditions come from collective agreements and therefore must be respected by both company and labor unions. The first model takes into account the saturation limit of the workstations, and the second model also includes the activation of the operators throughout the working day. Two computational experiments were carried out using a case study of the Nissan motor plant in Barcelona with two main objectives: (1) to study the repercussions of the saturation limit on the decrease in productivity on the line and (2) to evaluate the recovery of productivity on the line via both activation of operators, while maintaining the same quality in working conditions achieved by limiting the saturation, and auxiliary processors. By results we state that saturation limitation leads an important increase of work overload, which means average economic losses of 28,731.8 Euros/day. However, the productivity reduction may be counteracted by the work pace factor increase, at certain moments of workday, and/or by the incorporation of auxiliary processors into the line.
\end{abstract}

Keywords: Sequencing; Mixed-product line; Work overload; Saturation; Activity factor; Manufacturing operations.

\section{Preliminaries}

Currently, many production systems exist in which the manufacture or assembly of an entire product (or a subcomponent of the product) is carried out on the production line. At the same time, the increasing market requirements demand that companies offer a wide range of products with different options. This situation is commonly found in the automotive industry in which different products are manufactured and although these products belong to the same family, they have variable characteristics that require different component consumption and resource use, such as different processing times of operations. Obviously, not all vehicles share the same type of motor, and not all are equipped with the same components.

Assembly lines in the automotive industry are a clear example of this type of mixedproduct lines, which are known as Mixed-model Assembly Line $(M M A L)$. In this type of 
lines, different components (seats, steering wheels, pedals, etc.) are incorporated into the vehicle body depending on the type of vehicle that is assembled at each moment. Therefore, these lines must be flexible and able to adapt to each type of product assembled in them without incurring excessive costs.

In this way, to increase flexibility and reduce costs in terms of both workforce and storage, the MMALs face two basic problems: (1) the balancing of the line, known in the literature as the Assembly Line Balancing Problem (ALBP) (Salveson, 1955), and about which many variants exist (Battaïa and Dolgui, 2013; Becker and Scholl, 2006); and (2) the sequencing of mixed products in production lines and workshops.

The latter issue can be classified according to the variability of processing times of the operations required to assemble the products. If the units have heterogeneous processing times, in the stages of the production process in a workshop, we are facing permutation problems such as Flow-Shop Problems (Bautista, Cano, Companys and Ribas, 2012; Pan and Ruíz 2013). When the processing time of any operation depends on the number of units it is convenient to sequence the units in batches of pieces. In this case the problems are known as Economic Lot Scheduling Problems (ELSP) (Elmaghraby, 1978; Raza and Akgunduz, 2008). Finally, when the processing times are homogeneous during the stages of the production process the aim is to establish a manufacturing order for the products (and this order must be maintained as much as possible). These problems appear in the supply chain of production systems governed by the Just In Time (JIT, Toyota) and Douki-Seisan (DS, Nissan) ideologies and they are known as Mixed-Model Sequencing Problems (MMSP) (Bautista and Cano, 2011; Boysen, Fliedner and Scholl, 2009a, b; Solnon, Cung, Nguyen and Artigues, 2008).

In turn, the $M M S P$ can be classified according to the optimization criterion that affects one or more elements of the production system.

a) Minimization of the stock level of products and components. This category contains the Product Rate Variation Problem (PRVP) proposed by Miltenburg (1989) and whose purpose is to minimize the variation of production rates; and the Output Rate Variation Problem (ORVP) proposed by Monden (1983) and whose aim is to minimize the variation in the component consumption rates.

b) Minimization of the work overload. The variation of the processing times of operations based on the type of product can cause sometimes the time assigned to a workstation is less than the processing time of the operation of a product. When this happens, the processor does not have sufficient time to complete the work on the assigned product and then work overload appears. Without extra effort, this situation ends up generating backlog. In this case, the objective is to minimize the uncompleted work, which is also known as work overload. One example of this type of problems is the Mixed-Model Sequencing Problem with Workload Minimization (MMSP-W) both the original version, Yano and Rachamadugu (1991), and its variants Bautista, Cano and Alfaro (2012a, b).

c) Minimization of the number of subsequences with special options. These problems are focused on avoiding blockages caused by products that require additional work 
by offering special options. This problem is known as the Car Sequencing Problem (CSP) and was first described by Parrello, Kabat and Wos (1986).

Obviously, the above problems have been extended or combined in many papers in the literature. For instance, Lin and Chu (2013, 2014) among others, minimize the manufacturing total cost of a mixed-product assembly line considering labor, warehouse capacity and order fulfillment rates. Giard and Jeunet (2010) consider a cost function that involves two elements: the cost associated with the utility workers and the cost of setup. Thus, they simultaneously try: (1) to minimize the number of auxiliary operators necessary to complete the work required and therefore they minimize the work overload (MMSP-W objective); and (2) to minimize the setup times between product models (CSP objective).

Following the idea of extending the models in the literature, in this article, we focus on the (b) category of problems. Therefore, we address the sequencing problem of mixed products in production lines with the objective of minimizing the work overload (i.e., MMSP-W: Mixed-Model Sequencing Problem with Workload Minimization).

Although this type of problem has been widely treated in the literature, there are few papers that consider specific aspects of human resources involved in the production system. Among these few works, Celano, Costa, Fichera and Perrone (2004) introduced the human resource into the sequencing problem of a mixed-model U-assembly line in order to evaluate human factor policies impact on the optimal solution of the problem.

However, the processors of line workstations, in addition of automated systems, usually contain operators (i.e. persons) and these are subjected to working conditions defined according to laws, rules, contracts and also negotiations between the company and the workers' representative. Indeed, these conditions affect certain job characteristics, such as the length of working days, the saturation and occupancy rates of the processors, and the normal activity level of the operators, the acceptable performance level, among others.

As a result of works by Bautista et al. (2012b) and Alfaro (2015) and the scarcity of works on the sequencing problem considering the human factor of the processors of the line and the effect of working conditions on the productivity, in this work we propose an extension of the MMSP-W. This extension incorporates working conditions that must be guaranteed to the workers of an assembly line in the automotive Sector.

Specifically, we incorporate through new constraints the fulfillment of the maximum saturation of an operator throughout his working day. These new constraints limit the relation between the time used by the operator to carry out his workload and the available time to work. For this reason, we also incorporate two possible measures to counteract the negative effect of saturation conditions on productivity. First, we incorporate into the model the activity concept according the work by Bautista, Alfaro and Batalla (2015a). In this way the activation of workers at certain times of their workday will reduce productivity losses due to the limits of saturation. Obviously, the activation level of operators will also fulfill the limits established by collective agreements. Secondly, we reinforce the line with auxiliary processors in order to complete the required work. 
This work is structured as follows. In Section 2, the MMSP-W problem and some of its variants, such as the reference models used in this paper, are described. In Section 3, working conditions agreed at Collective Agreements are analyzed. Specifically, the focus is on the Work Schedule, the Workday and the processors' saturation. Section 4 is dedicated to incorporate the saturation conditions into the $M M S P-W$. For this purpose, it is necessary previously to distinguish between static and dynamic saturation. This section ends with the formulation of the two equivalent models, the $M 3 \cup 4 \_\eta$ and $M 4 \cup 3 \_\eta$. Section 5 is dedicated to an illustrative example. In Section 6, the new models are evaluated through a case study linked to an assembly line of engines of Nissan motor plant in Barcelona. This computational experience measures the impact of the saturation limitations in the stations regarding the increase of global work overload. In Section 7, we propose a series of corrective measures designed to reduce the global uncompleted work. Besides, two of these corrective measures, i.e., the activation of the processors and the incorporation of auxiliary processors, are described in greater detail in Section 8 and 9. As a result of the natural extension of the $M 3 \cup 4 \_\eta$ and $M 4 \cup 3 \_\eta$ models, considering the activation measure, in Section 8 other two new models are formulated the $M 3 \cup 4 \_\dot{\alpha} \_\eta$ and $M 4 \cup 3 \_\dot{\alpha} \_\eta$ model. In Section 10, we evaluate the results and the profits and losses obtained by the proposed models and the measures proposed to increase productivity, using the same data as in Section 5, in order to achieve a balance between productivity and ideal working conditions. Finally, we dedicate Section 11 to the conclusions and proposals for future work.

\section{The $M M S P-W$. Reference models}

The $M M S P-W$ consists of establishing the manufacturing order of $T$ units, which are grouped into a set $I$ of product types, in an assembly line composed of a set $K$ of workstations arranged in series. Each unit of product type $i(i=1, \ldots,|I|)$ requires from each homogeneous processor (operator, robot...) of a workstation $k(k=1, \ldots,|K|)$ a processing time $p_{i, k}$ measured at normal activity $\left(\alpha^{N}\right)$, but each processor has a normal working time known as cycle time $(c)$. This time is the standard time in which each processor is available to work on a product unit. Occasionally, to complete the work on a unit, the processor of workstation $k$ can retain that unit for a time longer than the cycle time. This time is referred to time window or temporal window $\left(l_{k}\right)$ and it fulfills $l_{k}-c>0$. However, using this time reduces the time available to work on the next unit of the sequence and finally, when the temporal window is not sufficient to complete the entire work required, the work overload is generated (see Fig. 1).

The objective of the problem is to minimize this work overload or maximize the completed work by taking into consideration the variation in operation processing times according to the product types; and assuming that the cycle time of the processors is determined from the average processing times of each type of product in regard with its presence in the demand plan. This objective can be achieved in two ways, first, by minimizing the incomplete work (work overload) by the processors; and second, by minimizing the downtime (idle time) of the processors.

Fig. 1 shows the effect produced by three sequences of six units of two types of products ( $A$ with high load and $B$ with low load) in a workstation. The first sequence $(A A A B B B)$ generates work overload, and the second sequence $(A A B B B A)$ generates idle time, whereas the third sequence $(A B A B A B)$ produces neither of this effects. 
Consideration of human resources in the Mixed-Model Sequencing Problem with Work Overload Minimization: Legal provisions and productivity improvement

J. Bautista, R. Alfaro-Pozo, C. Batalla-García
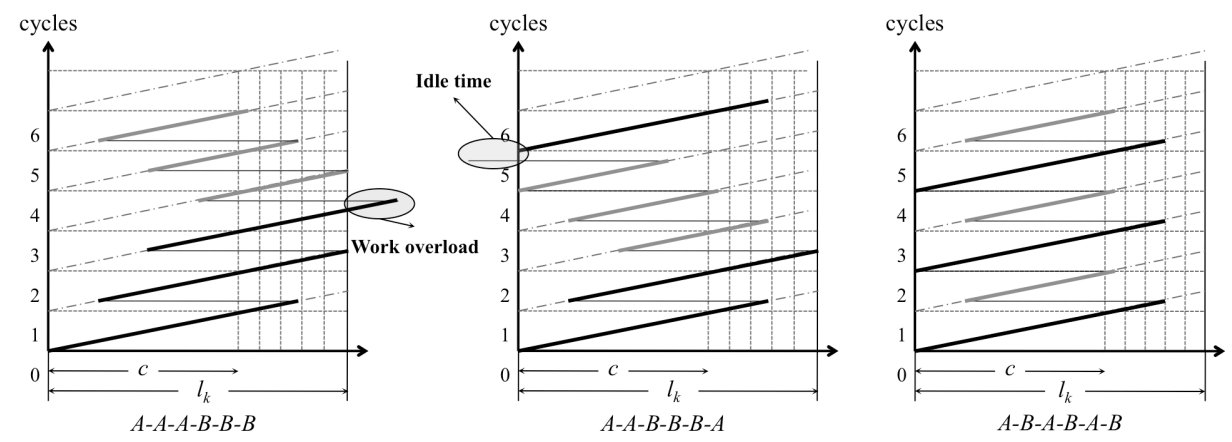

Fig. 1. Work overload, idle time, and completed work as a function of the sequence.

From the model proposed by Yano and Rachamadugu (1991), M1, and the model proposed by Scholl, Klein and Domschke (1998), M2, many variants of the MMSP-W have been studied. Indeed, from these models in which no links between stations are contemplated, Bautista and Suárez (2009) formulated two new models, i.e., M3 and M4, with links between stations and the assignment of a maximum time equal to $l_{k}$ for all units of products. Next, Bautista, Cano and Alfaro (2011) extended the M4 model to obtaining the $M 4^{\prime}$ model, which besides to minimizing the work overload, uses relative start instants and considers the temporal window in all of the workstations for all products and links between workstations; this model takes into account that a workstation can be viewed as more than a homogenous processor. Finally, Bautista et al. (2012b) formulated two new equivalent models, the $M 3 \cup 4$ model and the $M 4 \cup 3$ model.

To summarize, Table 1 lists the differences between the models mentioned above. To that end, we use the following characteristics: (1) the objective function with the minimization of the work overload $(W)$ or the maximization of the completed work $(V)$; (2) the variables associated with the start instants of the operations on absolute $\left(s_{k, t}\right)$ and relative $\left(\hat{s}_{k, t}\right)$ scales; (3) variables associated with the completed processing times, which are: the applied processing time at normal activity $\left(v_{k, t}\right)$ and the generated work overload $\left(w_{k, t}\right)$; (4) the maximum time that the processors of workstations can work on the last product unit $(t=T)$; (5) the range of the number of processors $\left(b_{k}\right)$ by workstation; (6) the consideration of links between serial stations.

\begin{tabular}{lccccccc}
\hline & $M 1$ & $M 2$ & $M 3$ & $M 4$ & $M 4^{\prime}$ & $M 3 \cup 4$ & $M 4 \cup 3$ \\
\hline Objective function & $\max V$ & $\min W$ & $\max V$ & $\min W$ & $\min W$ & $\max V / \min W$ & $\max V / \min W$ \\
Start instants & $s_{k, t}$ & $\hat{s}_{k, t}$ & $s_{k, t}$ & $\hat{s}_{k, t}$ & $\hat{s}_{k, t}$ & $s_{k, t}$ & $\hat{s}_{k, t}$ \\
Process variables & $v_{k, t}$ & $w_{k, t}$ & $v_{k, t}$ & $w_{k, t}$ & $w_{k, t}$ & $v_{k, t}, w_{k, t}$ & $v_{k, t}, w_{k, t}$ \\
Temporal window $t=T$ & $l_{k} \forall k$ & $c \forall k$ & $l_{k} \forall k$ & $l_{k} \forall k$ & $l_{k} \forall k$ & $l_{k} \forall k$ & $l_{k} \forall k$ \\
Range for $b_{k}$ & $b_{k} \geq 1$ & $b_{k}=1$ & $b_{k} \geq 1$ & $b_{k}=1$ & $b_{k} \geq 1$ & $b_{k} \geq 1$ & $b_{k} \geq 1$ \\
Station links & No & No & Yes & Yes & Yes & Yes & Yes \\
\hline
\end{tabular}

Table 1. Comparative study of the literature models.

Note that $M 3$ and $M 4$ models are an extension from the models proposed by Yano and Rachamadugu (1991) and Scholl et al. (1998). Likewise, the M3 44 and M4 $\cup 3$ models are an extension from $M 3$ and $M 4$, respectively. Thus, for this paper we use the $M 3 \cup 4$ and M4 $\cup 3$ models (Bautista et al., 2012b) as reference for the new models, which will 
Consideration of human resources in the Mixed-Model Sequencing Problem with Work Overload Minimization: Legal provisions and productivity improvement

J. Bautista, R. Alfaro-Pozo, C. Batalla-García

contemplate working conditions such as the level of occupation or saturation of the processors and the workers' activity.

\section{Working conditions established by law}

When a production system involves human resources, there is a set of legal provisions that must be met. These conditions imposed by laws, regulations or collective agreements refer to many labor issues, which affect the workers, such as ergonomics in the workplace, workers' rights, etc. (Llovera, Bautista, Llovera and Alfaro, 2014).

In our case, we analyze the legal provisions relating to the work schedule and the daily work organization in order to adapt the reference model for the MMSP-W (Bautista et al., 2012a, b) to real situations where human resources are present.

\subsection{The work schedule and the workday}

The duration of working day is decided by means of collective agreements or work contracts, according to the Article 34 of the Statute of the Workers' Rights. Thus, after analyzing various collective agreements in the automotive industry and especially from Nissan Motor Ibérica (NMISA), we can state that the daily working time usually is $8 \mathrm{~h}$ during $225-227$ days per year. However, these eight presence hours are reduced to a minimum limit for useful time of work of $6 \mathrm{~h}$ and $15 \mathrm{~min}$ and a maximum limit of $6 \mathrm{~h}$ and 59 min (Llovera et al., 2014). This reduction of effective working time is due to a number of rest periods that are determined by Statute of the Workers Rights and other legal provisions, such as collective agreements.

Finally, when the effective hours of the workday (HWD) and the number of units to manufacture in a day $(T)$ are known, the time given to any workstation to process any operation, i.e., the cycle time (c), is determined. Obviously, $c=H W D / T$.

\subsection{Daily work organization. Saturation of the processors}

In the work organization scope, three basic aspects are considered: work simplification and improvement of methods and industrial or administrative proceedings; analysis and determination of the work performance; and the selection of the correct workforce.

These aspects, in addition to meeting the general principle of adaptation of work for the person, must respect the conditions of the collective agreements in this regard. Thus, once established the operative methods and the permissible levels of quality in the manufacturing processes, the correct workloads and the performance or saturation levels are determined.

The saturation or performance is the ratio between the actual time used by an operator to carry out his workload and the available time that the operator has for performing that work. Its required value is one of the conditions that the major automotive companies negotiate with the workers' representatives. Indeed, European companies usually set limits for the maximum saturation $\left(\eta_{\max }^{\infty}\right)$ and the average saturation $\left(\eta_{\text {med }}^{\infty}\right)$ in the basis of their 
time scales and, therefore, the establishment of operator workloads throughout the workday must respect these saturation limits.

The maximum saturation is defined as the proportion of time used by the operation (workload) with the longest processing time regarding the available cycle time at a certain time of the workday. On the other hand, the average saturation is the proportion between all work required (in time units) with respect to all time available to complete that work. In Nissan's case, the usual limits for the maximum and average saturation are: $\eta_{\max }^{\infty}=1.2$ and $\eta_{m e d}^{\infty}=0.95$ respectively (regarding the centesimal time scale of the Methods Time Measurement system, 100/133MTM).

It should be noted that each company establishes its required and allowable performance levels by means of its collective agreements. For example, the SEAT S.A. collective agreement set these values in 1.0 and 0.96 respectively (considering the 75/100MTM system).

Obviously, these saturation conditions, will limit the amount of work completed by the operator. Indeed, a maximum average saturation equal to 0.95 means that the amount of work that an operator can perform in an effective workday of $6 \mathrm{~h}$ and $15 \mathrm{~min}$ is equal to $5 \mathrm{~h}$ and $56 \mathrm{~min}$, at normal activity.

Therefore, it is important to consider the saturation limits for the daily production schedule.

\section{Incorporating saturation limits into the MMSP-W}

\subsection{Previous definitions}

Before incorporating the saturation conditions into the mixed-model sequencing problem, it is necessary to distinguish between static and dynamic saturation.

The static saturation is determined by a cycle time $(c)$, a matrix of the processing times (at normal activity) of the operations (P: $\left.\left(p_{i, k}\right): i \in I, k \in K\right)$, and a demand plan $\left(\vec{d}=\left(d_{1}, \ldots, d_{|I|}\right)\right)$ composed of $T$ units of mixed products.

Specifically, the average static saturation, $\eta_{\text {med }}^{\circ}(k, c, \vec{d}, \mathrm{P})$, is associated with the workstation $k \in K$, the cycle time $(c)$, the demand plan $(\vec{d})$, and the set of processing times $(\mathrm{P})$. In this way, knowing that the average static saturation is the proportion of time required by each processor to complete the required work with respect to the time available, we have:

$$
\eta_{\text {med }}^{\circ}(k, c, \vec{d}, P)=\frac{1}{c \cdot T} \cdot \sum_{i=1}^{|I|} p_{i, k} \cdot d_{i} \quad(k=1, \ldots,|K|)
$$

Similarly, the maximum static saturation, $\eta_{\max }^{\circ}(k, c, \mathrm{P})$, is associated with the workstation $k \in K$, the cycle time $(c)$, and the set of processing times (P). Thus, considering that the maximum static saturation is the proportion of time that each 
processor requires to complete the most laborious operation with respect to the cycle time, we define:

$$
\eta_{\max }^{\circ}(k, c, P)=\frac{1}{c} \cdot \max _{i \in I}\left\{p_{i, k}\right\} \quad(k=1, \ldots,|K|)
$$

Consequently, if we take into consideration the conditions established between the company and the workers' representative, the following equations should be satisfied:

$$
\begin{array}{ll}
\eta_{\text {med }}^{\circ}(k, c, \vec{d}, P) \leq \eta_{\text {med }}^{\infty} & (k=1, \ldots,|K|) \\
\eta_{\text {max }}^{\circ}(k, c, P) \leq \eta_{\text {max }}^{\infty} & (k=1, \ldots,|K|)
\end{array}
$$

On the other hand, the dynamic saturation of the workstations depends on the manufacturing sequence of the products $\left(\pi(T)=\pi_{1}, \ldots, \pi_{T}\right)$, the cycle time $(c)$, and a matrix of times (at normal activity) corresponding to the work completed $\left(\mathrm{V}=\left(v_{k, t}\right): k \in\right.$ $K, T=1, \ldots, T)$ at each workstation and position in the sequence. The average and maximum dynamic saturation gives the following equations:

$$
\begin{array}{ll}
\eta_{\text {med }}(k, c, \pi(T), V)=\frac{1}{c \cdot T} \cdot \sum_{t=1}^{T} v_{k, t} & (k=1, \ldots,|K|) \\
\eta_{\max }(k, c, \pi(T), V)=\frac{1}{c} \cdot \max _{1 \leq t \leq T}\left\{v_{k, t}\right\} & (k=1, \ldots,|K|)
\end{array}
$$

Therefore, to limit the average and maximum dynamic saturation in the line workstations, we add to the $M M S P$ - $W$ models the following restrictions:

$$
\begin{array}{lr}
\sum_{t=1}^{T} v_{k, t} \leq \eta_{\text {med }}^{\infty} \cdot c \cdot T & (k=1, \ldots,|K|) \\
v_{k, t} \leq \eta_{\text {max }}^{\infty} \cdot c & (k=1, \ldots,|K| ; t=1, \ldots, T)
\end{array}
$$

As shown, the static saturation depends on the required work time and the dynamic saturation depends on the completed work time. Therefore, it is possible saturation levels are above the limit values set by collective agreements, in case of a demand plan with a highly demanding load or an inappropriate fabrication sequence.

It should be noted that the violation of the maximum saturation is considered unacceptable and requires review by the department of times and measures in order to search for alternatives in the assembly process that will reduce processing times. 
However, this situation does not occur with average saturation. Indeed, when the average saturation, either static or dynamic, exceeds the admissible $\eta_{m e d}^{\infty}$ limit value in certain workstations, the processors do not have sufficient time to complete the required work. Therefore, the work overload will increase against the reduction of the $\eta_{m e d}^{\infty}$ value. Indeed, it is possible to determine the static work overload associated with each processor of the workstation $k \in K$ as follows:

$$
w_{0}\left(k, c, \vec{d}, P, \eta_{m e d}^{\infty}\right)=c \cdot T \cdot \max \left\{0, \eta_{m e d}^{\circ}(k, c, \vec{d}, P)-\eta_{m e d}^{\infty}\right\} \quad(k=1, \ldots,|K|)
$$

And considering all workstations of the line, it is possible to estimate the static work overload of the line by means of the adding of static work overloads of workstations multiplied by their processors $\vec{b}=\left(b_{1}, \ldots, b_{|K|}\right)$ :

$$
W_{0}\left(c, \vec{d}, \vec{b}, P, \eta_{m e d}^{\infty}\right)=\sum_{k=1}^{|K|} b_{k} \cdot w_{0}\left(k, c, \vec{d}, P, \eta_{m e d}^{\infty}\right)
$$

On the other hand, the dynamic work overloads associated with the workstations and the line are determined in the following manner:

$$
\begin{gathered}
w\left(k, c, \pi(T), V, \eta_{\text {med }}^{\infty}\right)=c \cdot T \cdot \max \left\{0, \eta_{\text {med }}(k, c, \pi(T), V)-\eta_{\text {med }}^{\infty}(k=1, \ldots,|K|)\right. \\
W\left(c, \vec{b}, \pi(T), V, \eta_{\text {med }}^{\infty}\right)=\sum_{k=1}^{|K|} b_{k} \cdot w\left(k, c, \pi(T), V, \eta_{\text {med }}^{\infty}\right)
\end{gathered}
$$

Obviously, the work overload value that derives from the dynamic saturation $(W)$ will always be equal or greater than the work overload derived from the static saturation $\left(W_{0}\right)$. This situation occurs because the effects produced by the variation of processing times in combination with the sequence must be added in the dynamic situation.

\section{2. $M M S P-W$ models with saturation constraints}

Taking into account the dynamic saturation definitions and the $M 3 \cup 4$ and $M 4 \cup 3$ models for the MMSP-W (Bautista et al., 2012b), whose difference is the time scale used to calculate the start instants of operations at workstations; we propose two new equivalents models. The new models, $M 3 \cup 4 \_\eta$ and $M 4 \cup 3 \_\eta$, consider the saturation conditions and their parameters and variables are the following:

Parameters:

$K \quad$ Set of workstations, arranged in series, that makes up the line $(k=1, \ldots,|K|)$

$b_{k} \quad$ Number of homogeneous processors at workstation $k(k=1, \ldots,|K|)$

I Set of product types that must be manufactured in the line $(i=1, \ldots,|I|)$ 
Consideration of human resources in the Mixed-Model Sequencing Problem with Work Overload Minimization: Legal provisions and productivity improvement

J. Bautista, R. Alfaro-Pozo, C. Batalla-García

$d_{i} \quad$ Programmed demand of the product type $i(i=1, \ldots,|I|)$

$p_{i, k} \quad$ Processing time (normal activity) required by one unit of a product type $i(i=1, \ldots,|I|)$ at workstation $k(k=1, \ldots,|K|)$ for each homogeneous processor

$T \quad$ Total demand. Obviously: $\sum_{i=1}^{|I|} d_{i}=T$

$t \quad$ Position index in the sequence $(t=1, \ldots, T)$

$c \quad$ Cycle time. Standard time assigned to each homogeneous processor in the workstations ( $k=$ $1, \ldots,|K|)$ to process any product unit

$l_{k} \quad$ Temporal window. Maximum time that each homogeneous processor of workstation $k(k=$ $1, \ldots,|K|)$ is allowed to work on any unit of product; once the cycle has been completed, the maximum time that a unit of product can be retained in station $k$ is $l_{k}-c>0$

$\eta_{m e d}^{\infty} \quad$ Allowable average saturation by the processors of workstations $(k=1, \ldots,|K|)$

$\eta_{\max }^{\infty} \quad$ Allowable maximum saturation by the processors of workstations $(k=1, \ldots,|K|)$

Variables:

$x_{i, t} \quad$ Binary variable equal to 1 if the product unit $i(i=1, \ldots,|I|)$ is assigned to the position $t(t=$ $1, \ldots, T)$ of the sequence and 0 otherwise

$s_{k, t} \quad$ Start instant for the $t^{t h}$ unit of the sequence of products at workstation $k(k=1, \ldots,|K|)$

$\hat{s}_{k, t} \quad$ Relative start instant. Positive difference between the start instant and the earliest start instant of the $t^{\text {th }}$ operation in the workstation $k(k=1, \ldots,|K|)$

It is fulfilled $\hat{s}_{k, t}=\left[s_{k, t}-(t+k-2) \cdot c\right]^{+}$

$v_{k, t} \quad$ Processing time applied by each homogeneous processor (at normal activity) to the $t^{\text {th }}$ product unit sequenced in the workstation $k(k=1, \ldots,|K|)$

$w_{k, t} \quad$ Work overload measured in units of time (at normal activity) generated by the $t^{\text {th }}$ product unit sequenced in each homogeneous processor of the workstation $k(k=1, \ldots,|K|)$

And the new mathematical models are the following:

$M 3 \cup 4 \_\eta$ model:

$$
\max V=\sum_{k=1}^{|K|}\left(b_{k} \cdot \sum_{t=1}^{T} v_{k, t}\right) \Leftrightarrow \min W=\sum_{k=1}^{|K|}\left(b_{k} \cdot \sum_{t=1}^{T} w_{k, t}\right)
$$

Subject to:

$$
\begin{array}{lc}
\sum_{t=1}^{T} x_{i, t}=d_{i} & i=1, \ldots,|I| \\
\sum_{i=1}^{|I|} x_{i, t}=1 & t=1, \ldots, T \\
v_{k, t}+w_{k, t}=\sum_{i=1}^{|I|} p_{i, k} \cdot x_{i, t} & k=1, \ldots,|K| ; t=1, \ldots, T
\end{array}
$$


J. Bautista, R. Alfaro-Pozo, C. Batalla-García

$$
\begin{array}{lc}
\sum_{t=1}^{T} v_{k, t} \leq \eta_{\text {med }}^{\infty} \cdot c \cdot T & k=1, \ldots,|K| \\
v_{k, t} \leq \eta_{\max }^{\infty} \cdot c & k=1, \ldots,|K| ; t=1, \ldots, T \\
s_{k, t} \geq(t+k-2) \cdot c & k=1, \ldots,|K| ; t=1, \ldots, T \\
s_{k, t} \geq s_{k, t-1}+v_{k, t-1} & k=1, \ldots,|K| ; t=2, \ldots, T \\
s_{k, t} \geq s_{k-1, t}+v_{k-1, t} & k=2, \ldots,|K| ; t=1, \ldots, T \\
s_{k, t}+v_{k, t} \leq(t+k-2) \cdot c+l_{k} & k=1, \ldots,|K| ; t=1, \ldots, T \\
s_{k, t}, v_{k, t}, w_{k, t} \geq 0 & k=1, \ldots,|K| ; t=1, \ldots, T \\
x_{i, t} \in\{0,1\} & i=1, \ldots,|I| ; t=1, \ldots, T
\end{array}
$$

$M 4 \cup 3 \_\eta$ model:

$$
\min W=\sum_{k=1}^{|K|}\left(b_{k} \cdot \sum_{t=1}^{T} w_{k, t}\right) \Leftrightarrow \max V=\sum_{k=1}^{|K|}\left(b_{k} \cdot \sum_{t=1}^{T} v_{k, t}\right)
$$

Subject to:

$$
\begin{array}{lc}
\sum_{t=1}^{T} x_{i, t}=d_{i} & i=1, \ldots,|I| \\
\sum_{i=1}^{|I|} x_{i, t}=1 & t=1, \ldots, T \\
v_{k, t}+w_{k, t}=\sum_{i=1}^{|I|} p_{i, k} \cdot x_{i, t} & k=1, \ldots,|K| ; t=1, \ldots, T \\
\sum_{t=1}^{T} v_{k, t} \leq \eta_{m e d}^{\infty} \cdot c \cdot T & \\
v_{k, t} \leq \eta_{\text {max }}^{\infty} \cdot c & k=1, \ldots,|K| \\
\hat{s}_{k, t} \geq \hat{s}_{k, t-1}+v_{k, t-1}-c & k=1, \ldots,|K| ; t=1, \ldots, T \\
\hat{s}_{k, t} \geq \hat{s}_{k-1, t}+v_{k-1, t}-c & k=1, \ldots,|K| ; t=2, \ldots, T \\
\hat{s}_{k, t}+v_{k, t} \leq l_{k} & k=2, \ldots,|K| ; t=1, \ldots, T \\
\hat{s}_{k, t}, v_{k, t}, w_{k, t} \geq 0 & k=1, \ldots,|K| ; t=1, \ldots, T \\
x_{i, t} \in\{0,1\} & k=1, \ldots,|K| ; t=1, \ldots, T \\
\hat{s}_{1,1}=0 & i=1, \ldots,|I| ; t=1, \ldots, T
\end{array}
$$


The objective functions, (13) and (25), are the same and denote the equivalence between the minimization of the total dynamic work overload $(W)$ and the maximization of the total completed work $(V)$. Constraints (14) and (26) represent the satisfaction of the programmed demand. Constraints (15) and (27) force the assignment of each product unit to one position of the sequence. The set of constraints (16) and (28) fix the relationships among the required processing time, the completed work, and the work overload by workstation and moment. Constraints (17) and (29) restrict the average saturation, and constraints (18) and (30) restrict the maximum saturation at workstations. The sets (19)- (22) determine the absolute start instants of the operations at the workstations; and the sets $(31)-(33)$ denote the relative start instants of the $t(t=1, \ldots, T)$ unit sequenced at workstation $k(k=1, \ldots,|K|)$. Constraints (23) and (34) establish the non-negativity of the variables. Constraints (24) and (35) establish as binary the assignment variables of units to the sequence. Finally, the equality (36) fixes the start instant of the operations.

\section{An illustrative exemple}

The following example is used to illustrate the models and concepts defined above.

There are six units of product $(T=6)$, of which three are type $A$, one is type $B$ and two are type $C$. The units are processed at three workstations $(|K|=3)$ with different numbers of processors $\left(b_{k}\right)$; the processing times of processors (at normal activity) for each type of unit $(A, B, C)$ at each station $\left(m_{1}, m_{2}, m_{3}\right)$ are listed in Table 2 . Furthermore, the cycle time is $c=4 \mathrm{~s}$, the time window is $l_{k}=6 \mathrm{~s}$, for all stations, and the limits for the static and dynamic maximum and medium saturations are $\eta_{\max }^{\infty}=1.32$ and $\eta_{\text {med }}^{\infty}=$ 1.00 , respectively.

\begin{tabular}{lccccc}
\hline$k$ & $A\left(d_{A}=3\right)$ & $B\left(d_{B}=1\right)$ & $C\left(d_{C}=2\right)$ & $b_{k}$ & \\
\hline$m_{1}$ & 5 & 4 & 3 & 1 & $\left(V_{0}\left(m_{1}\right)=25\right)$ \\
$m_{2}$ & 5 & 4 & 4 & 2 & $\left(V_{0}\left(m_{2}\right)=54\right)$ \\
$m_{3}$ & 4 & 3 & 5 & 1 & $\left(V_{0}\left(m_{3}\right)=25\right)$ \\
\hline Total & $19\left(V_{0}(A)=57\right)$ & $15\left(V_{0}(B)=15\right)$ & $16\left(V_{0}(C)=32\right)$ & & $V_{0}=104$ \\
\hline
\end{tabular}

Table 2. Number of homogeneous processors $\left(b_{k}\right)$ by station $(k \in K)$, and processing times $\left(p_{i, k}\right)$, at normal activity $\left(\alpha^{N}=1\right)$, by product type $(i \in I)$ and processor from each workstation.

If we calculate the static saturations that are generated by the demand plan, we observe (see Fig. 2) how the established limits for the average saturation are not fulfilled, being the static saturations greater than the allowable value $\left(\eta_{m e d}^{\infty}=1.00\right)$. However, this does not happen with the maximum static saturation, whose values are below the set limit $\left(\eta_{\max }^{\infty}=\right.$ 1.32). 


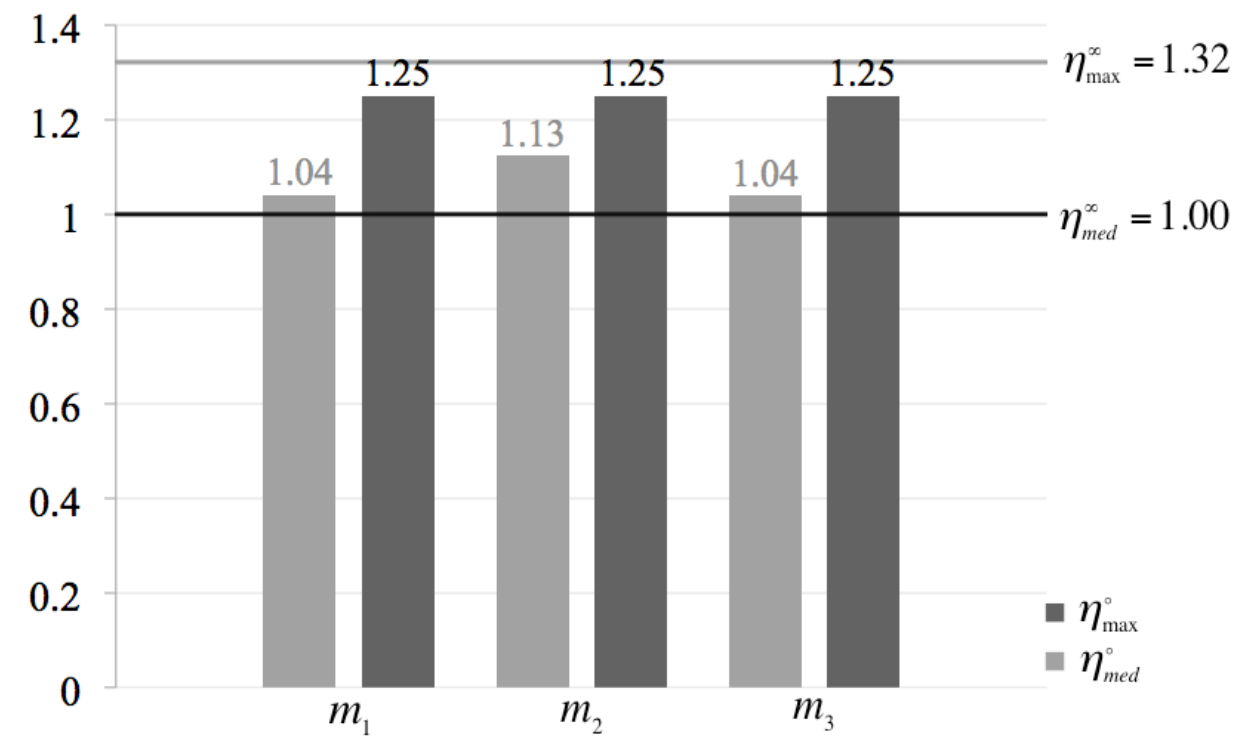

Fig. 2. Maximum and average static saturation by workstation generated by the demand plan and the line features.

The static average saturation results denote an unavoidable work overload of the line if the established limits are imposed. Indeed, by means of Eqs. (9) and (10), it is possible to calculate the said unavoidable value of non-completed work: $W_{0}=8 \mathrm{~s}$. This indicates how the incorporation of saturation constraints into the problem may suppose a work overload increase and therefore a productivity decrement.

Next, the results obtained by the reference models and those proposed with saturation conditions are summarized (Table 3 ).

\begin{tabular}{lccccccc}
\hline & Sequence & $V$ & $W$ & $w\left(m_{1}\right)$ & $w\left(m_{2}\right)$ & $w\left(m_{3}\right)$ & $s_{|K|, T}+v_{|K|, T}$ \\
\hline$M 3 \cup 4$ & $C_{1}-A_{1}-C_{2}-A_{2}-B_{1}-A_{3}$ & 101 & 3 & 1 & 2 & 0 & 34 \\
$M 4 \cup 3$ & $C_{1}-C_{2}-B_{1}-A_{1}-A_{2}-A_{3}$ & 101 & 3 & 1 & 2 & 0 & 34 \\
$M 3 \cup 4 \_\eta$ & $C_{1}-A_{1}-B_{1}-A_{2}-C_{2}-A_{3}$ & 96 & 8 & 1 & 6 & 1 & 34 \\
$M 4 \cup 3 \_\eta$ & $C_{1}-B_{1}-A_{1}-C_{2}-A_{2}-A_{3}$ & 96 & 8 & 1 & 6 & 1 & 34 \\
\hline
\end{tabular}

Table 3. Summary of the example's results given by models $M 3 \cup 4, M 4 \cup 3, M 3 \cup 4 \_\eta$ and $M 4 \cup 3 \_\eta$.

This table (Table 3) together with Fig. 3 allows to us to conclude the following:

- Both pairs of models, without and with saturation conditions respectively, offer the same results except for the product sequence.

- The models without saturation conditions give better results in regard with the work overload. However these models suppose an average dynamic saturation for the processors of workstations superior to the allowable values.

- The incorporation of saturation conditions into the sequencing models allows for complying with the established limit in regard with the workers' occupation. Nevertheless this supposes an increase of the non-completed work. 
- By fulfilling saturations set through collective agreements may mean a worse line productivity.
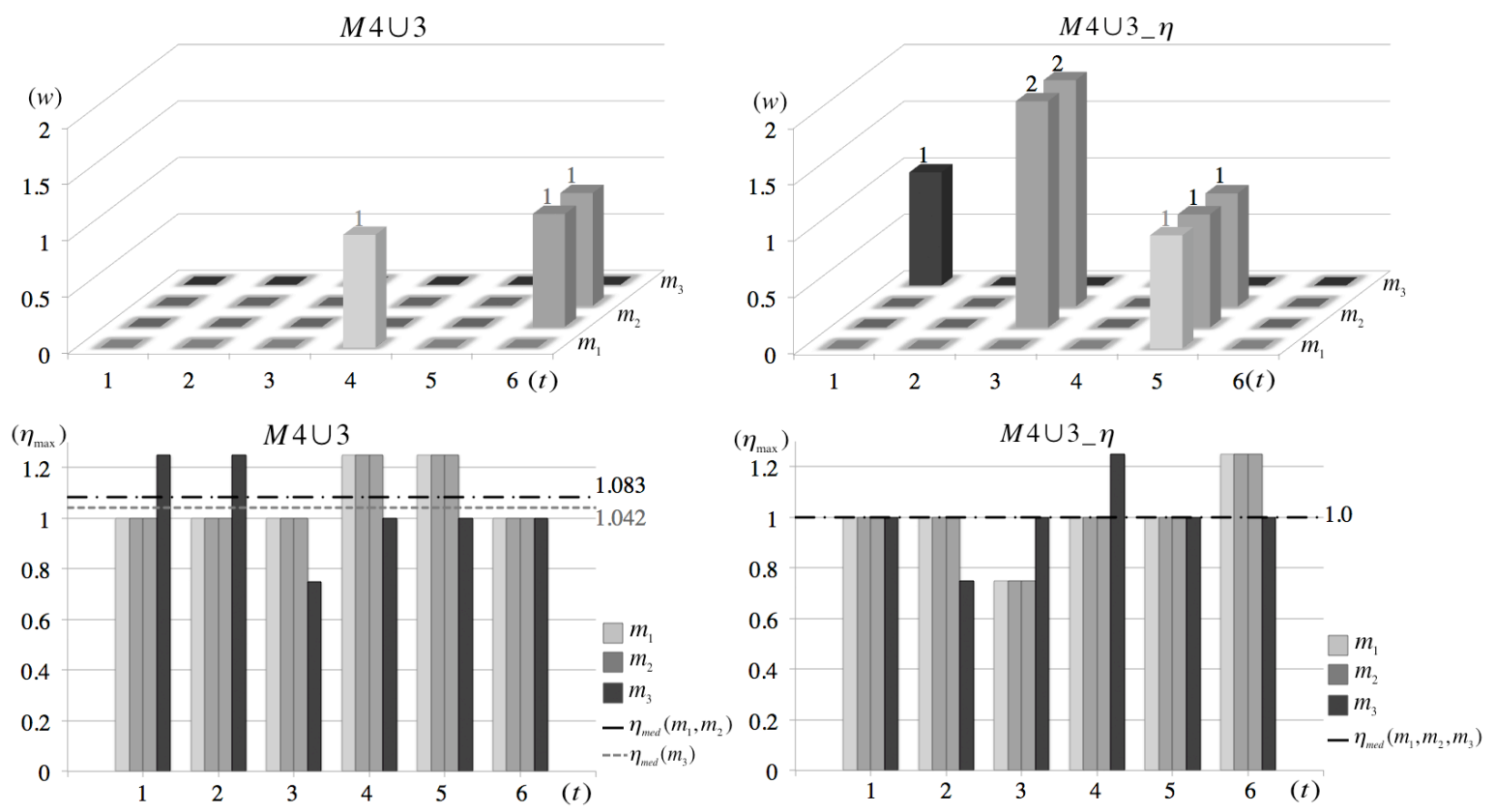

Fig. 3. Work overload and maximum and average dynamic saturation given by $M 4 \cup 3$ and $M 4 \cup 3 \_\eta$ models.

\section{Saturation condition effects in the $M M S P-W$. Case study.}

Next, we evaluate the effect of the saturation limitation on the increase in work overload using a case study linked to the assembly line of engines at Nissan in Barcelona.

Specifically, we will run the $M 3 \cup 4 \_\eta$ and $M 4 \cup 3 \_\eta$ models for a set $E$ of 23 different demand plans (see Block I of Table 7 in Bautista et al., 2012b) in order to obtaining sequences with minimum work overload. All data instances consist of a workday divided into two shifts of $8 h$ which means a total of 13.125 effective hours of work per day, after discount the statutory breaks and rest periods.

All plans must satisfy the same total demand of engines, $T=270$, which is divided into nine types of engines, $|I|=9$. These types are grouped into three families according to the vehicle types: (1) $4 \times 4$ vehicles $\left(p_{1}, p_{2}, p_{3}\right),(2)$ vans $\left(p_{4}, p_{5}\right)$, and (3) average tonnage trucks $\left(p_{6}, \ldots, p_{9}\right)$. The different engines have different processing time of operations at workstations, $p_{i, k}(i=1, \ldots,|I| ; k=1, \ldots,|K|)$.

All engines must be assembled in a line with 21 serial workstations $(|K|=21)$, all of them with one homogeneous processor $\left(b_{k}=1, \forall k \in K\right)$ that is equivalent to a team of two workers with identical skills and tools and the same requirements for auxiliary equipment. Moreover, all processors of workstations get the same cycle time, $c=175 \mathrm{~s}$, and an identical time window that is equal to $l_{k}=195 \mathrm{~s}$ that supposes a slack over the $10 \%$ of cycle time to work on a product unit in the sequence at any workstation. 
Finally, regarding saturation limits, we use the values established by the collective agreement of Nissan, that is, $\eta_{\max }^{\infty}=1.20$ for the maximum saturation and $\eta_{\text {med }}^{\infty}=0.95$ for the average saturation.

Considering the above data set, we run the models through the LP Solver of the Gurobi Optimizer 4.5.0 in an Apple Macintosh iMac computer with an Intel Core i7 2.93-GHz processor, $8 \mathrm{~GB}$ of RAM memory, and a MAC OS X 10.6,7 operating system with a CPU time limit for each production plan to $7200 \mathrm{~s}$.

Before running the models, the values for the maximum and average static saturation are calculated for each demand plan, according to Eqs. (1) and (2). Thus, we can check whether all production plans fulfill the maximum value of static saturation permitted per workstation and therefore all of them are technically feasible on the line. Furthermore, we can also calculate, from Eq. (10), the inevitable overload generated at stations with an average static saturation higher than the allowed by Nissan $\left(\eta_{m e d}^{\infty}=0.95\right)$.

Table 4 shows the calculated results and these obtained after running the $M 3 \cup 4 \_\eta$ and $M 4 \cup 3 \_\eta$ models. Indeed, the first column of the table represents the number of the production plan $(\varepsilon \in \mathrm{E})$. The second column shows the set of workstations that contain oversaturated processors when only the static saturation is considered. The third column shows the values of the global work overload $\left(W_{3 \cup 4}, W_{4 \cup 3}\right)$ achieved by the reference models, M3 $\cup 4$ and M4 3 (Bautista et al., 2012a). The fourth column shows the inevitable overload of the line due to the limit of the average static saturation $\left(W_{0 \_}\right)$ calculated from Eq. (10). The fifth column presents the values of the dynamic work overload of the line obtained by the new models $\left(W_{3 \cup 4 \_\eta}=W_{4 \cup 3 \_\eta}\right)$. Finally, the sixth column shows the work overload increase because of the incorporation of the saturation constraints in both models $\left(R P D_{1}, R P D_{2}\right)$; these values are calculated by the following equations:

$$
\begin{array}{lr}
R P D_{1}(\varepsilon)=\frac{W_{3 \cup 4 \_\eta}(\varepsilon)-W_{3 \cup 4}(\varepsilon)}{W_{3 \cup 4}(\varepsilon)} \cdot 100 & (\varepsilon=\# 1, \ldots, \#|\mathrm{E}|) \\
R P D_{2}(\varepsilon)=\frac{W_{4 \cup 3 \_\eta}(\varepsilon)-W_{4 \cup 3}(\varepsilon)}{W_{4 \cup 3}(\varepsilon)} \cdot 100 & (\varepsilon=\# 1, \ldots, \#|\mathrm{E}|)
\end{array}
$$

Based on obtained results (Table 4), we observe the following:

1. None of the 23 demand plans violates the limitation of both static and dynamic maximum saturation $\left(\eta_{\max }^{\infty}=1.20\right)$ imposed on all workstations by collective agreement. Therefore, both models can find a solution.

2. Workstations $4,9,10,16,17$ and 18 present an average static saturation higher than the admissible in the 23 production plans. However, the rest of workstations are never oversaturated, which means that none of them fails to the limit imposed on the medium static saturation $\left(\eta_{\text {med }}^{\infty}=0.95\right)$.

3. The incorporation of the saturation constraints supposes a considerable increase of work overload. Indeed, taking only the static situation into account $\left(W_{0 \_}\right)$, the 
work overload values get worse, on average, until to reach $12,570.2 \mathrm{~s}$. This value turns into a number of uncompleted engines of 72 engines per day, considering the cycle time.

4. Both models, $M 3 \cup 4 \_\eta$ and $M 4 \cup 3 \_\eta$, offer the same solutions for all instances. In addition, in all plans, the static work overload of the line $\left(W_{0_{-} \eta}\right)$ coincides with the values of dynamic work overload given by the models $\left(W_{3 \cup 4 \_\eta}=W_{4 \cup 3 \_\eta}\right)$. This indicates the following three observations: (1) the optimal values (maximums) for the completed work are reached in the 23 plans; (2) none of the 23 sequences of manufacture generates idle time; and (3) the effect of saturation on the work overload of workstations causes the sequence does not influence the results.

5. The increase in work overload values $\left(R P D_{1}, R P D_{2}\right)$ given by $M 3 \cup 4 \_\eta$ model, by limiting the average saturation of stations ranges from $986.3 \%(\varepsilon=\# 10)$ to $7,047.0 \%(\varepsilon=\# 11)$, with an average value of $2,930.3 \%$. Meanwhile, the $M 4 \cup 3 \_\eta$ model, with relative start instants, reaches increases of $986.3 \%(\varepsilon=$ $\# 10), 15,018.6 \%$ ( $\varepsilon=\# 11$ ), and 4,143.0\%, on average.

6. The improvement in working conditions by the average saturation constraints at workstations can involve a daily work loss whose average value is equivalent to 72 engines with a range of 9 engines because of the quantity of uncompleted work. Without this limitation, the daily work loss is equivalent to an average of 3 engines with range of 7 engines.

7. Taking into account production line of engines supposes a consolidated operation profit of $10 \%$ over the total value of a motor $(4,000 €)$, i.e., the loss of an engine means a cost for the line of $400 €$, we can calculate the daily economic losses. Indeed, the economic losses due to the limitation of the average saturation are equivalent to $28,731.8$ Euros/day, on average, with a range of 3,589.7 Euros/day; without the limitation, the average economic loss because of work overload is1,064.7 Euros/day, with a range of 2,582.9 Euros/day.

\begin{tabular}{rccccccc}
\hline$\varepsilon$ & $k \in K: \eta_{\text {med }}^{\circ}(k)>\eta_{\text {med }}^{\infty}$ & $W_{3 \cup 4}$ & $W_{4 \cup 3}$ & $W_{0_{-} \eta}$ & $W_{3 \cup 4 \_\eta}=W_{4 \cup 3_{-} \eta}$ & $R P D_{1}$ & $R P D_{2}$ \\
\hline$\# 1$ & $4,9,10,16,17,18$ & 251.0 & 187.0 & 12315.0 & $12315.0^{*}$ & 4806.4 & 6485.6 \\
$\# 2$ & $4,9,10,16,17,18$ & 444.0 & 341.0 & 12458.0 & $12458.0^{*}$ & 2705.9 & 3553.4 \\
$\# 3$ & $4,9,10,11,16,17,18,21$ & 477.0 & 427.0 & 12210.0 & $12210.0^{*}$ & 2459.7 & 2759.5 \\
$\# 4$ & $4,9,10,16,17,18$ & 402.0 & 310.0 & 12470.0 & $12470.0^{*}$ & 3002.0 & 3922.6 \\
$\# 5$ & $4,9,10,16,17,18,21$ & 754.0 & 633.0 & 13012.5 & $13012.5^{*}$ & 1625.8 & 1955.7 \\
$\# 6$ & $4,9,10,16,17,18$ & 525.0 & 413.0 & 12910.0 & $12910.0^{*}$ & 2359.0 & 3025.9 \\
$\# 7$ & $4,9,10,16,17,18,21$ & 818.0 & 742.0 & 12722.5 & $12722.5^{*}$ & 1455.3 & 1614.6 \\
$\# 8$ & $4,9,10,16,17,18$ & 228.0 & 139.0 & 12018.0 & $12018.0^{*}$ & 5171.1 & 8546.0 \\
$\# 9$ & $4,9,10,16,17,18$ & 824.0 & 732.0 & 13363.0 & $13363.0^{*}$ & 1521.7 & 1725.5 \\
$\# 10$ & $4,9,10,11,16,17,18,21$ & $1208.0^{*}$ & $1208.0^{*}$ & 13122.0 & $13122.0^{*}$ & 986.3 & 986.3 \\
$\# 11$ & $4,9,10,11,16,17,18$ & 165.0 & 78.0 & 11792.5 & $11792.5^{*}$ & 7047.0 & 15018.6 \\
$\# 12$ & $4,9,10,16,17,18$ & 406.0 & 284.0 & 12246.0 & $12246.0^{*}$ & 2916.3 & 4212.0 \\
$\# 13$ & $4,9,10,16,17,18$ & 383.0 & 286.0 & 12551.0 & $12551.0^{*}$ & 3177.0 & 4288.5 \\
$\# 14$ & $4,9,10,16,17,18$ & 500.0 & 420.0 & 12646.0 & $12646.0^{*}$ & 2429.2 & 2911.0 \\
$\# 15$ & $4,9,10,16,17,18,21$ & 506.0 & 433.0 & 12393.5 & $12393.5^{*}$ & 2349.3 & 2762.2 \\
\hline
\end{tabular}


Consideration of human resources in the Mixed-Model Sequencing Problem with Work Overload Minimization: Legal provisions and productivity improvement

J. Bautista, R. Alfaro-Pozo, C. Batalla-García

\begin{tabular}{cccccccc}
\hline$\# 16$ & $4,9,10,16,17,18$ & 321.0 & 227.0 & 12363.0 & $12363.0^{*}$ & 3751.4 & 5346.3 \\
$\# 17$ & $4,9,10,16,17,18,21$ & 550.0 & 478.0 & 12597.5 & $12597.5^{*}$ & 2190.5 & 2535.5 \\
$\# 18$ & $4,9,10,16,17,18$ & 673.0 & 605.0 & 13208.0 & $13208.0^{*}$ & 1862.6 & 2083.1 \\
$\# 19$ & $4,9,10,11,16,17,18,21$ & 949.0 & $945.0^{*}$ & 12810.0 & $12810.0^{*}$ & 1249.8 & 1255.6 \\
$\# 20$ & $4,9,10,16,17,18$ & 233.0 & 139.0 & 11875.0 & $11875.0^{*}$ & 4996.6 & 8443.2 \\
$\# 21$ & $4,9,10,16,17,18$ & 652.0 & 560.0 & 13065.0 & $13065.0^{*}$ & 1903.8 & 2233.0 \\
$\# 22$ & $4,9,10,16,17,18,21$ & 1006.0 & 987.0 & 13062.5 & $13062.5^{*}$ & 1198.5 & 1223.5 \\
$\# 23$ & $4,9,10,11,16,17,18$ & 188.0 & 140.0 & 11902.5 & $11902.5^{*}$ & 6231.1 & 8401.8 \\
\hline & Average values & 541.9 & 465.8 & 12570.2 & 12570.2 & 2930.3 & 4143.0 \\
\hline
\end{tabular}

Table 4. Oversaturated workstations $(k \in K)$, work overload $\left(W_{3 \cup 4}, W_{4 \cup 3}, W_{0 \_}, W_{3 \cup 4 \_\eta}, W_{4 \cup 3 \_\eta}\right)$, and percentage increase $\left(R P D_{1}, R P D_{2}\right)$ of the dynamic work overload obtained by limiting saturation for 23 demand plans $(\varepsilon \in \mathrm{E})$.

Obviously, the limitation of the occupation level of workers gets worse the productivity of the assembly line. However, thanks to these saturation constraints, the legal conditions imposed by collective agreements are fulfilled and therefore the working conditions are improved.

\section{Measures used to reduce the work overload}

The consideration of specific aspects of real production systems, such as the saturation limitation of workers, may result in an increase in work overload and therefore in an increase in economic losses because of the uncompleted work.

Consequently, keeping in mind the main objective of $M M S P-W$ and the obligation to respect the saturation limits established in the collective agreements, the following alternatives are proposed to counter the violation of the saturation limits, and thus, to improve working conditions without damaging the productivity of the assembly line.

1. Resort to methods and times department to search for alternatives in the assembly process in order to reduce the processing times of operations. This action is not immediate because it requires the intervention of the product and process engineering.

2. Increase the activation level of the processors (based on activity factor) like Bautista, Alfaro and Batalla made in their work (Bautista et al., 2015a). In this way, the work assigned to each processor will be carried out in less time, and thus, the ratio between the total available time and the real work time will be reduced. In others words, this action would reduce the average saturation. Indeed, given a demand plan, the average saturation of a processor will be higher or lower depending on whether the activity factor of this processor is lower or higher, respectively. Obviously, this activation may not exceed the limits established by the collective agreements at any time of the workday.

3. Strengthen the production line to increase its capacity by incorporating auxiliary processors, either multi-skilled or not, in the system. These auxiliary processors could assist the oversaturated workstations. 
4. Resort to rotation between consecutive stations. Using this approach, the oversaturated workstations may be offset, in the medium term, with lower saturations of other workstations with higher ergonomic quality. It should be noted that this measure is not permitted in companies that belong to the Organization for Economic Cooperation and Development $(O C D E)$ because it is not allow exceeding the average saturation limit at any time of day and any workstation. However, this measure could be considered for those countries where no such limit exists.

In this work, we focus on the second and third measures proposed to reduce the overload.

\section{Incorporating the processors' activation}

In order to reduce production losses generated by limiting saturation processors and based on the work published by Bautista et al., (2015a, b), a series of concepts regarding the variation of the activity or work pace of the operators are described. Similarly, new mathematical models that incorporate the possibility of increasing the work pace of processors to reduce the workload while respecting saturation limits established by the collective agreement are formulated.

\subsection{Processing times and work pace factor}

Normally the automotive sector uses timing techniques, tables/estimates and MTM (Methods Time Measurement) systems as time measuring instruments. These techniques allow assigning a predetermined o standard time to any operation or manual method through the decomposition of the operation into basic movements.

In this regard, once the standard processing times are established, according to the MTM system, the normal processing times $\left(p_{i, k}: i \in I, k \in K\right)$ must be established, considering the normal activity set by the company by means of collective agreements. This normal activity corresponds to the required work pace with which workers must perform their workload, in normal conditions.

In Nissan's case these normal processing times are established as the resultant of the direct application of the MTM_100 times (standard times with centesimal scale) with an activity of 110 (normal work pace set by Nissan):

$$
p_{i, k}\left(M T M \_110\right)=p_{i, k}\left(M T M \_100\right) \cdot \frac{100}{110} \quad \forall i \in I, \forall k \in K
$$

where $p_{i, k}=p_{i, k}\left(M T M \_110\right): i \in I, k \in K$ are the processing times (pre-set) required by products to the processors of workstations, when processors work at normal activity, according Nissan's case.

However, analyzing the collective agreement of SEAT S.A., the normal processing times are established from the standard times MTM_75 (normal activity according the scale 
75/100), considering that normal activity set by SEAT S.A. is 96 (MTM_96). Thus, the Eq. (39) is the following for SEAT S.A.:

$$
p_{i, k}\left(\text { MTM_96) }=p_{i, k}\left(M T M_{-} 75\right) \cdot \frac{75}{96} \quad \forall i \in I, \forall k \in K\right.
$$

In this way, defining the work pace factor by product and workstation, $\alpha_{i, k}$ ( $i \in I, k \in$ $K)$ as the ratio between a given activity and the normal activity on the same timescale, it is possible to change the processing times with normal activity, $p_{i, k}$, to another one, $\hat{p}_{i, k}$, regardless of the timescale used.

$$
\alpha_{i, k} \cdot \hat{p}_{i, k}=\alpha^{N} \cdot p_{i, k}: \text { if } \alpha^{N}=1 \rightarrow \alpha_{i, k}=p_{i, k} / \hat{p}_{i, k} \quad \forall i \in I, \forall k \in K
$$

Obviously, if activity increases above de normal $\left(\alpha^{N}\right)$, the processing times of operations will be reduced. In contrast, if the work pace of an operator is lower than the normal, the processing times will be longer. In this way, if an operator works with a greater work pace than the normal, he will take less time to perform his workload, his saturation level will be less and the work overload will be reduced.

It must be noted that automotive industries and labor unions sometimes also agree a maximum or optimal activity level over the activity established as normal through collective agreements. This optimal activity corresponds to the $120 \%$ of the normal activity, in the case of Nissan, and is the maximum work pace which an operator can withstand without harming its health.

Summarizing, taking into account the work pace levels that can be defined in the collective agreements, we differentiate between the standard, the normal and the optimal activities; being the standard, the activity considered as normal by the time scale and with which the standard times are pre-determined; being the normal activity, the activity level required by company; and being the optimal activity, the maximum work pace allowed.

Obviously, each company will adopt its values established. For instance, in regard with the Nissan's Collective Agreement, we can associate to the standard, normal and optimal activities, the scale times MTM_100, MTM_110 and MTM_132 respectively, which correspond to activity factors $\alpha_{i, k}^{\circ}=0.90, \alpha_{i, k}^{N}=1.0$ and $\alpha_{i, k}^{*}=1.2$.

\subsection{The work pace throughout the workday}

Bautista et al., (2015a) established a direct correlation between the activity factor and the stress level over time, taking into consideration firstly, the idea from Robert Yerkes and John Dodson, who said that when stress increases up to a certain optimum point of stress, the operators' performance improves; and, secondly, the idea that worker's performance varies throughout the working day, and therefore, the relationship between the operators' performance and their level of "activation", which is reflected by their level of stress, follows a concave function (Muse, Harris and Field, 2003). Thanks to this correlation the authors defined, first, a stepped function for the work pace factor throughout time (Bautista, 
Alfaro, Batalla and Cano, 2014) and, afterwards, three additional functions, the triangular, the trapezoidal and the parabolic one (Bautista et al., 2015a).

In this way, the authors were able to reduce the work overload by reducing the processing times of operations by means of the increase of dynamic activity factor at specific instants of the workday in accordance with the defined functions.

For this reason, this paper is based on the activation functions defined by Bautista et al. (2015a) to reduce the great work overload generated by the saturation conditions.

Specifically, we incorporate the dynamic activity factor into the $M 3 \cup 4 \_\eta$ and $M 4 \cup 3 \_\eta$ models, following the procedure used by Bautista et al., (2015a) to formulate the models $M 3 \cup 4 \_\dot{\alpha} I$ and $M 4 \cup 3 \_\dot{\alpha} I$.

\subsection{The $M M S P$ - $W$ with saturation constraints and activity factor variation}

From the $M 3 \cup 4 \_\eta$ and $M 4 \cup 3 \_\eta$ models and considering the variation of work pace factor of operators throughout their workday (Bautista et al., 2015a), we propose the $M 3 \cup 4 \_\dot{\alpha} I_{-} \eta$ and $M 4 \cup 3 \_\dot{\alpha} I_{-} \eta$ models. The additional parameters and variables of the new models are:

Parameters:

$\dot{\alpha}_{k, t} \quad$ Dynamic factor of the work pace or activity associated with the $t^{\text {th }}$ operation of the product sequence $(t=1, \ldots, T)$ at the workstation $k(k=1, \ldots,|K|)$. Note that $\alpha_{i, k}$ is the activity factor by product and workstation and it not depends on the sequence.

$\dot{\alpha}_{t} \quad$ Dynamic factor of the work pace or activity associated with the period $t(t=1, \ldots, T+|K|-1)$ of the extended workday. This extended workday includes $T$ manufacturing cycles (total demand) and $|K|-1$ additional cycles, which are required to complete the required work by the production units in all the workstations. Note that if we associate the same dynamic factor with each moment of the workday in all of the workstations, we will have:

$$
\dot{\alpha}_{k, t}=\dot{\alpha}_{t+k-1} \quad(k=1, \ldots,|K| ; t=1, \ldots, T)
$$

Variables:
$\hat{v}_{k, t} \quad$ Processing time reduced by the dynamic activity factor $\dot{\alpha}_{k, t}$. It is established that:
$v_{k, t}=\dot{\alpha}_{k, t} \cdot \hat{v}_{k, t} \quad(k=1, \ldots,|K| ; t=1, \ldots, T)$

Hence, considering the equality $\dot{\alpha}_{k, t}=\dot{\alpha}_{t+k-1}(k=1, \ldots,|K| ; t=1, \ldots, T)$ and the parameters and variables defined above, the new models for the $M M S P-W$ are:

$M 3 \cup 4 \_\dot{\alpha} I \_\eta$ model:

$$
\max V=\sum_{k=1}^{|K|}\left(b_{k} \cdot \sum_{t=1}^{T} v_{k, t}\right) \Leftrightarrow \min W=\sum_{k=1}^{|K|}\left(b_{k} \cdot \sum_{t=1}^{T} w_{k, t}\right)
$$

Subject to: constraints (14)-(16) from $M 3 \cup 4 \_\eta$ model

$$
\dot{\alpha}_{t+k-1} \cdot \hat{v}_{k, t}-v_{k, t}=0 \quad k=1, \ldots,|K| ; t=1, \ldots, T
$$


J. Bautista, R. Alfaro-Pozo, C. Batalla-García

$$
\begin{array}{lc}
\sum_{t=1}^{T} \hat{v}_{k, t} \leq \eta_{\text {med }}^{\infty} \cdot c \cdot T & k=1, \ldots,|K| \\
\hat{v}_{k, t} \leq \eta_{\max }^{\infty} \cdot c & k=1, \ldots,|K| ; t=1, \ldots, T \\
s_{k, t} \geq(t+k-2) \cdot c & k=1, \ldots,|K| ; t=1, \ldots, T \\
s_{k, t} \geq s_{k, t-1}+\hat{v}_{k, t-1} & k=1, \ldots,|K| ; t=2, \ldots, T \\
s_{k, t} \geq s_{k-1, t}+\hat{v}_{k-1, t} & k=2, \ldots,|K| ; t=1, \ldots, T \\
s_{k, t}+\hat{v}_{k, t} \leq(t+k-2) \cdot c+l_{k} & k=1, \ldots,|K| ; t=1, \ldots, T \\
s_{k, t}, v_{k, t}, \hat{v}_{k, t}, w_{k, t} \geq 0 & k=1, \ldots,|K| ; t=1, \ldots, T
\end{array}
$$

$M 4 \cup 3 \_\dot{\alpha} I \_\eta$ model:

$$
\min W=\sum_{k=1}^{|K|}\left(b_{k} \cdot \sum_{t=1}^{T} w_{k, t}\right) \Leftrightarrow \max V=\sum_{k=1}^{|K|}\left(b_{k} \cdot \sum_{t=1}^{T} v_{k, t}\right)
$$

Subject to: constraints (26) $-(28)$ from $M 4 \cup 3 \_\eta$ model

$$
\begin{array}{lc}
\dot{\alpha}_{t+k-1} \cdot \hat{v}_{k, t}-v_{k, t}=0 & k=1, \ldots,|K| ; t=1, \ldots, T \\
\sum_{t=1}^{|T|} \hat{v}_{k, t} \leq \eta_{m e d}^{\infty} \cdot c \cdot T & k=1, \ldots,|K| \\
\hat{v}_{k, t} \leq \eta_{\max }^{\infty} \cdot c & k=1, \ldots,|K| ; t=1, \ldots, T \\
\hat{s}_{k, t} \geq \hat{s}_{k, t-1}+\hat{v}_{k, t-1}-c & k=1, \ldots,|K| ; t=2, \ldots, T \\
\hat{s}_{k, t} \geq \hat{s}_{k-1, t}+\hat{v}_{k-1, t}-c & k=2, \ldots,|K| ; t=1, \ldots, T \\
\hat{s}_{k, t}+\hat{v}_{k, t} \leq l_{k} & k=1, \ldots,|K| ; t=1, \ldots, T \\
\hat{s}_{k, t}, v_{k, t}, \hat{v}_{k, t}, w_{k, t} \geq 0 & k=1, \ldots,|K| ; t=1, \ldots, T
\end{array}
$$

The new set of constraints (45) and (54) serves to reduce or lengthen the applied processing times depending on the work pace factor. The remainder constraints coincide with the $M 3 \cup 4 \_\eta$ and $M 4 \cup 3 \_\eta$ models, leaving out both the constraints that limit the saturation of the processors, i.e., (46) - (47) and (55) - (56), and the ones that determine the start instants of the operations, i.e., (48) - (51) and (57) - (59), that now consider the reduced processing time applied, $\hat{v}_{k, t}$. Obviously, if we consider $\dot{\alpha}_{t}=1, \forall t$, the $M 3 \mathrm{U}$ 4_$\dot{\alpha} I \_\eta$ and $M 4 \cup 3 \_\dot{\alpha} I \_\eta$ models are equivalent to the $M 3 \cup 4 \_\eta$ and $M 4 \cup 3 \_\eta$ models, respectively.

On the other hand, in terms of average and maximum static saturations, it is now necessary to consider the matrix of the static activity factors $\left(A:=\left(\alpha_{i, k}\right): i \in I, k \in K\right)$ : 


$$
\begin{aligned}
\eta_{\text {med }}^{\circ}(k, c, \vec{d}, P, A) & =\frac{1}{c \cdot T} \cdot \sum_{i=1}^{|I|} \frac{p_{i, k}}{\alpha_{i, k}} \cdot d_{i} & (k=1, \ldots,|K|) \\
\eta_{\text {max }}^{\circ}(k, c, P, A) & =\frac{1}{c} \cdot \max _{i \in I}\left\{\frac{p_{i, k}}{\alpha_{i, k}}\right\} & (k=1, \ldots,|K|)
\end{aligned}
$$

Therefore, the unavoidable static work overload supported by each processor of workstation $k \in K$ has the following form:

$$
w_{0}\left(k, c, \vec{d}, \mathrm{P}, \mathrm{A}, \eta_{\text {med }}^{\infty}\right)=c \cdot T \cdot \max \left\{0, \eta_{\text {med }}^{\circ}(k, c, \vec{d}, \mathrm{P}, \mathrm{A})-\eta_{m e d}^{\infty}\right\} \quad(k=1, \ldots,|K|)
$$

And the static work overload of the line is calculated in the following manner:

$$
W_{0}\left(c, \vec{d}, \vec{b}, \mathrm{P}, \mathrm{A}, \eta_{\text {med }}^{\infty}\right)=\sum_{k=1}^{|K|} b_{k} \cdot w_{0}\left(k, c, \vec{d}, \mathrm{P}, \mathrm{A}, \eta_{\text {med }}^{\infty}\right)
$$

Obviously, for practical purposes, for determining both the maximum and average static saturation of the processors of workstations, given a production plan $(\vec{d})$ and the processing times of operations $(\mathrm{P})$, the set of activity factor values $(\mathrm{A})$ will be independent of the workstations $(k \in K)$ and products $(i \in I)$. Therefore, the average activity factor, determined from the corresponding values at every moment of the working day, will be used.

On the other hand, if we take into consideration the dynamic activity factors $(\dot{\mathrm{A}}:=$ $\left.\left(\dot{\alpha}_{k, t}\right): k \in K, t=1, \ldots, T\right)$ and the manufacturing sequence $\pi(T)=\left\{\pi_{1}, \ldots, \pi_{T}\right\}$, the equations that, respectively, determine the dynamic saturation (medium and maximum) and the dynamic work overload (elemental and global) are the following:

$$
\begin{array}{cc}
\eta_{\text {med }}(k, c, \pi(T), \mathrm{V}, \dot{\mathrm{A}})=\frac{1}{c \cdot T} \cdot \sum_{t=1}^{|T|} \frac{v_{k, t}}{\dot{\alpha}_{k, t}} & (k=1, \ldots,|K|) \\
\eta_{\max }(k, c, \pi(T), \mathrm{V}, \dot{\mathrm{A}})=\frac{1}{c} \cdot \max _{1 \leq t \leq T}\left\{\frac{v_{k, t}}{\dot{\alpha}_{k, t}}\right\} & (k=1, \ldots,|K|) \\
w\left(k, c, \pi(T), \mathrm{V}, \dot{\mathrm{A}}, \eta_{m e d}^{\infty}\right)=c \cdot T \cdot \max \left\{0, \eta_{m e d}(k, c, \pi(T), \mathrm{V}, \dot{\mathrm{A}})-\eta_{m e d}^{\infty}\right\} & (k=1, \ldots,|K|) \\
W\left(c, \vec{b}, \pi(T), \mathrm{V}, \dot{\mathrm{A}}, \eta_{m e d}^{\infty}\right) & =\sum_{k=1}^{|K|} b_{k} \cdot w\left(k, c, \pi(T), \mathrm{V}, \dot{\mathrm{A}}, \eta_{m e d}^{\infty}\right)
\end{array}
$$

Note that equations represent the general case. In our particular case study, we have considered that operators perform their workload with the same work pace regardless of the workstation in which they find themselves; that is: $\dot{\alpha}_{k, t}=\dot{\alpha}_{t+k-1}(k=1, \ldots,|K| ; t=$ $1, \ldots, T)$. 


\section{Increased line capacity by auxiliary processors}

An alternative measure to deal with the increased work overload produced by saturation conditions is to incorporate reinforcement operators into the line. Really, once known the inevitable work overload per station, both static and dynamic, it is possible to determine the number of auxiliary workers needed to complete this unfinished work. This calculation can be done by two ways:

I. Auxiliary processors required by the line: from the obtained overall work overload, both static and dynamic, and the total available time by processor throughout the effective shift work, it is possible to determine a lower bound of the number of auxiliary processors necessary to eliminate the work overload. For that matter the following points should be noted:

a. The auxiliary processors are multi-skilled and therefore they can perform suitably any task assigned to any workstation.

b. The movement of processors between stations is considered negligible.

c. There are not more workstations overloaded simultaneously than the number of auxiliary processors.

In such conditions, the number of auxiliary multi-skilled processors needed to eliminate the static and dynamic work overload respectively, is calculated as follows:

$$
\begin{aligned}
\Delta b^{\circ} & =\sum_{k=1}^{|K|} b_{k} \cdot \max \left\{0, \eta_{\text {med }}^{\circ}(k, c, \vec{d}, \mathrm{P}, \mathrm{A})-\eta_{\text {med }}^{\infty}\right\} \\
\Delta b & =\sum_{k=1}^{|K|} b_{k} \cdot \max \left\{0, \eta_{\text {med }}(k, c, \vec{d}, \mathrm{~V}, \dot{\mathrm{A}})-\eta_{\text {med }}^{\infty}\right\}
\end{aligned}
$$

Obviously, this number of multi-skilled processors can be determinate for a specific workstation:

$$
\begin{array}{ll}
\Delta b_{k}^{\circ}=\max \left\{0, \eta_{\text {med }}^{\circ}(k, c, \vec{d}, P, A)-\eta_{\text {med }}^{\infty}\right\} & (k=1, \ldots,|K|) \\
\Delta b_{k}=\max \left\{0, \eta_{\text {med }}(k, c, \vec{d}, V, \dot{A})-\eta_{\text {med }}^{\infty}\right\} & (k=1, \ldots,|K|)
\end{array}
$$

Fulfilling the following:

$$
\begin{aligned}
\Delta b^{\circ} & =\sum_{k=1}^{|K|} b_{k} \cdot \Delta b_{k}^{\circ} \\
\Delta b & =\sum_{k=1}^{|K|} b_{k} \cdot \Delta b_{k}
\end{aligned}
$$


II. Auxiliary processors by workstation: from the work overload generated at each station and the total available time by processor throughout the effective shift work, an upper bound, of the number of processors necessary to complete the required work by workstation, can be obtained. In this way, it must be noted:

a. The auxiliary processors are specialized in operations assigned to a single station and, therefore, they are assigned exclusively to one workstation. That is, each workstation overloaded receives individualized assistance of a specialized processor.

Accordingly, the number of specialized processors necessary to eliminate the work overload generated by the saturation conditions, both static and dynamic, is determined as follows:

$$
\begin{aligned}
\widehat{\Delta} b^{\circ} & =\sum_{k=1}^{|K|}\left\lceil b_{k} \cdot \Delta b_{k}^{\circ}\right\rceil \\
\widehat{\Delta} b & =\sum_{k=1}^{|K|}\left\lceil b_{k} \cdot \Delta b_{k}\right\rceil
\end{aligned}
$$

Along these lines, we consider two opposite ways to counteract the negative effect of limiting the processors' saturation. First, we propose multi-skilled processors, that can provide assistance to any workstations and, secondly, we suggest specialized processors that are assigned to one specific station.

It should be noted that, so far, we have only consider the calculation of auxiliary processors. However, in our case study, each processor consists of a team of two workers. For this reason, we denote as $\mathrm{H}$ the number of operators that compose one processor and therefore we define the auxiliary operator functions as follows:

$$
\begin{gathered}
\Delta H^{\circ}=\left\lceil H \cdot \Delta b^{\circ}\right\rceil \\
\Delta H=\lceil H \cdot \Delta b\rceil \\
\widehat{\Delta} H^{\circ}=\sum_{k=1}^{|K|}\left\lceil H \cdot b_{k} \cdot \Delta b_{k}^{\circ}\right\rceil \\
\widehat{\Delta} H=\sum_{k=1}^{|K|}\left\lceil H \cdot b_{k} \cdot \Delta b_{k}\right\rceil
\end{gathered}
$$

Where $\Delta H^{\circ}$ and $\Delta H$ is the number of multi-skilled workers necessary to eliminate the work overload generated in the line by the static and dynamic saturation conditions respectively; and $\widehat{\Delta} H^{\circ}$ and $\hat{\Delta} H$ is the number of specialized workers necessary to counteract the negative effect of the static and dynamic saturation conditions respectively. It should be 
noted that if $H=1$ the equations (73)-(76) will be equivalent to equations (77)-(80) respectively.

\section{Effect of activation and saturation conditions. Case study}

Next, we determine the inevitable static work overload of the line and the dynamic work overload according to the $M 3 \cup 4 \_\dot{\alpha} I_{-} \eta$ and $M 4 \cup 3 \_\dot{\alpha} I_{-} \eta$ models. We achieve this goal by working from the same case corresponding to the Nissan engine plant in Barcelona that was used in the exploitation of $M 3 \cup 4 \_\eta$ and $M 4 \cup 3 \_\eta$ models but considering simultaneously the limitation of saturation and the activation of the processors.

In this experiment, a number of features of the assembly line have been taken into account to define the stepped $\left(\dot{\alpha}^{S}\right)$, triangular $\left(\dot{\alpha}^{T}\right)$, trapezoidal $\left(\dot{\alpha}^{Z}\right)$ and parabolic $\left(\dot{\alpha}^{P}\right)$ functions of the dynamic factor of activity. We state the following:

- Factor of normal activity and maximum allowed: $\alpha^{N}=1.0, \alpha^{\max }=1.1$. It should be noted that the considered maximum value is lower than the optimal value established by the collective agreement $\left(\alpha^{*}=1.2\right)$.

- Shift 1: $t_{0}=45, t_{\text {med }}=67, t_{\infty}=90$.

- Shift 2: $t_{0}=180, t_{\text {med }}=202, t_{\infty}=225$.

- Average of the activity factor: $\overline{\alpha^{S}}=1.0 \hat{3} ; \overline{\alpha^{T}}=1.05 ; \overline{\alpha^{Z}}=\overline{\alpha^{P}}=1.0 \hat{6}$.

In this way, all workers will synchronously perform their work, period by period, throughout the workday and will follow the work pace set by the activity factor imposed by the functions defined in Bautista et al. (2015a) and their respective functions with average value. For illustration see the Figure 4 (Fig. 4) that corresponds to the stepped function $\left(\dot{\alpha}^{S}\right)$.

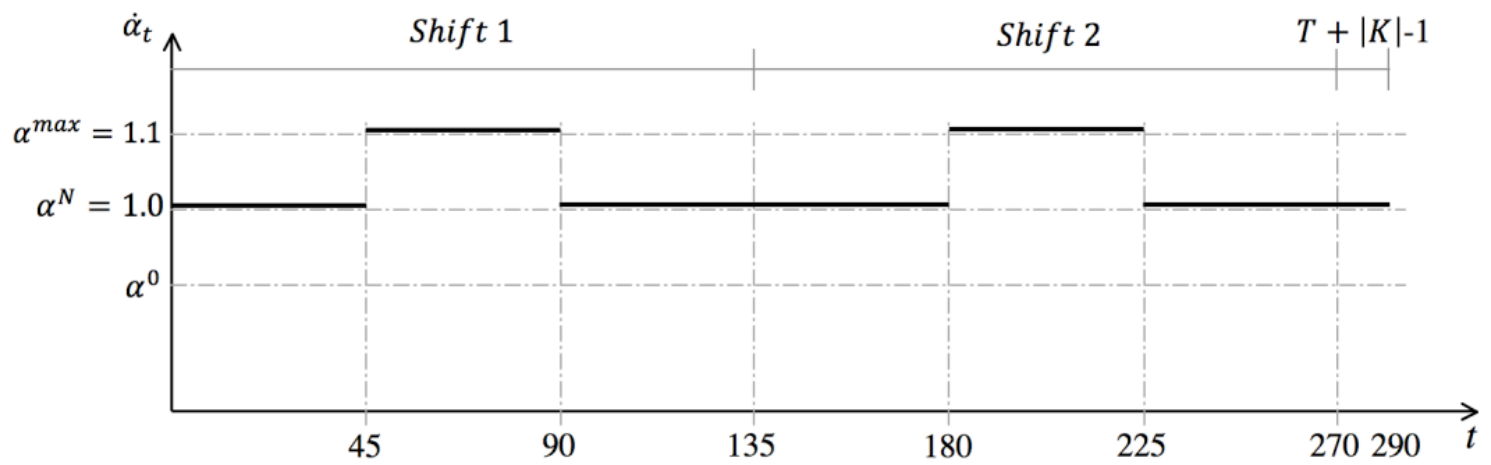

Fig 4. Stepped function for the work pace factor in the NISSAN case.

The work overload results achieved in this experiment are shown in Table 5. The first column enumerates the 23 plans of production $(\varepsilon=\# 1, \ldots, \# 23)$. The following four columns determine the inevitable work overload $\left(W_{0}\right)$ obtained by each instance when the static saturation limitations are considered. Specifically, there are shown the values obtained without considering activation of processors (second column) and the values obtained when 
an average activation is considering according to the average values of the four functions for work pace of workers.

Furthermore, the last six columns represent the values obtained with the $M 3 \cup 4 \_\dot{\alpha} I \_\eta$ and $M 4 \cup 3 \_\dot{\alpha} I \_\eta$ models when all cases for the workers' activation are considered. Indeed, the sixth column shows the work overload given by $M 3 \cup 4 \_\eta$ and $M 4 \cup 3 \_\eta$ models, that is equal to consider a work pace function equal to the normal activity factor all along the workday.

\begin{tabular}{|c|c|c|c|c|c|c|c|c|c|c|}
\hline \multirow{2}{*}{$\varepsilon$} & \multicolumn{4}{|c|}{$W_{0}\left(\alpha_{i, k}, d_{i}\right)$} & \multirow{2}{*}{$W_{3 \cup 4 \_\eta}, W_{4 \cup 3 \_\eta}$} & \multicolumn{5}{|c|}{$W_{3 \cup 4_{-} \dot{\alpha} I_{-} \eta}=W_{4 \mathrm{U} 3_{-} \dot{\alpha} I_{-} \eta}$} \\
\hline & - & $\overline{\alpha^{S}}$ & $\overline{\alpha^{T}}$ & $\overline{\alpha^{Z}}=\overline{\alpha^{P}}$ & & $\dot{\alpha}^{S}$ & $\dot{\alpha}^{T}$ & $\overline{\alpha^{S}}$ & $\overline{\alpha^{T}}$ & $\dot{\alpha}^{Z}=\dot{\alpha}^{P}$ \\
\hline \#1 & $12,315.0$ & $4,220.6$ & 591.1 & 0.0 & $12,315.0$ & $4,601.8$ & 796.0 & 4361.3 & 620.6 & 0.0 \\
\hline \#2 & $12,458.0$ & $4,312.6$ & 725.2 & 0.0 & $12,458.0$ & $4,692.7$ & 884.9 & 4456.3 & 761.5 & 0.0 \\
\hline \#3 & $12,210.0$ & $4,123.9$ & 794.6 & 0.0 & $12,210.0$ & $4,509.2$ & 895.4 & 4261.3 & 834.4 & 0.0 \\
\hline$\# 4$ & $12,470.0$ & $4,269.0$ & 638.7 & 0.0 & $12,470.0$ & $4,649.5$ & 843.2 & 4411.3 & 670.6 & 0.0 \\
\hline \#5 & $13,012.5$ & $4,549.7$ & $1,053.8$ & 0.0 & $13,012.5$ & $4,929.8$ & $1,202.9$ & 4701.3 & $1,106.5$ & 0.0 \\
\hline \#6 & $12,910.0$ & $4,506.1$ & 872.0 & 0.0 & $12,910.0$ & $4,882.2$ & $1,072.5$ & 4656.3 & 915.6 & 0.0 \\
\hline \#7 & $12,722.5$ & $4,361.0$ & $1,080.4$ & 0.0 & $12,722.5$ & $4,743.9$ & $1,212.2$ & 4506.3 & $1,134.4$ & 0.0 \\
\hline$\# 8$ & $12,018.0$ & $4,075.5$ & 448.2 & 0.0 & $12,018.0$ & $4,459.6$ & 657.1 & 4211.3 & 470.6 & 0.0 \\
\hline \#9 & $13,363.0$ & $4,694.8$ & $1,071.9$ & 0.0 & $13,363.0$ & $5,066.8$ & $1,258.2$ & 4851.3 & $1,125.5$ & 0.0 \\
\hline$\# 10$ & $13,122.0$ & $4,404.5$ & $1,545.1$ & 0.0 & $13,122.0$ & $4,795.6$ & $1,660.4$ & 4551.3 & $1,622.4$ & 0.0 \\
\hline \#11 & $11,792.5$ & $3,838.4$ & 355.7 & 0.0 & $11,792.5$ & $4,231.1$ & 520.8 & 3966.3 & 373.5 & 0.0 \\
\hline$\# 12$ & $12,246.0$ & $4,209.0$ & 649.0 & 0.0 & $12,246.0$ & $4,590.8$ & 801.1 & 4349.3 & 681.5 & 0.0 \\
\hline \#13 & $12,551.0$ & $4,335.8$ & 704.4 & 0.0 & $12,551.0$ & $4,715.1$ & 907.0 & 4480.3 & 739.6 & 0.0 \\
\hline \#14 & $12,646.0$ & $4,398.7$ & 789.0 & 0.0 & $12,646.0$ & $4,777.3$ & 968.3 & 4545.3 & 828.5 & 0.0 \\
\hline \#15 & $12,393.5$ & $4,271.9$ & 796.7 & 0.0 & $12,393.5$ & $4,653.1$ & 946.6 & 4414.3 & 836.5 & 0.0 \\
\hline \#16 & $12,363.0$ & $4,249.7$ & 619.6 & 0.0 & $12,363.0$ & $4,630.4$ & 823.9 & 4391.3 & 650.6 & 0.0 \\
\hline \#17 & $12,597.5$ & $4,375.5$ & 872.9 & 0.0 & $12,597.5$ & $4,755.0$ & $1,022.3$ & 4521.3 & 916.5 & 0.0 \\
\hline \#18 & $13,208.0$ & $4,646.4$ & $1,010.1$ & 0.0 & $13,208.0$ & $5,020.7$ & $1,210.2$ & 4801.3 & $1,060.6$ & 0.0 \\
\hline \#19 & $12,810.0$ & $4,312.6$ & $1,294.6$ & 0.0 & $12,810.0$ & $4,700.6$ & $1,403.5$ & 4456.3 & $1,359.4$ & 0.0 \\
\hline \#20 & $11,875.0$ & $3,983.5$ & 440.5 & 0.0 & $11,875.0$ & $4,370.0$ & 599.7 & 4116.3 & 462.5 & 0.0 \\
\hline \#21 & $13,065.0$ & $4,554.5$ & 919.6 & 0.0 & $13,065.0$ & $4,929.1$ & $1,120.4$ & 4706.3 & 965.6 & 0.0 \\
\hline \#22 & $13,062.5$ & $4,452.9$ & $1,330.8$ & 0.0 & $13,062.5$ & $4,838.9$ & $1,440.5$ & 4601.3 & $1,397.4$ & 0.0 \\
\hline \multirow[t]{2}{*}{$\# 23$} & $11,902.5$ & $3,935.2$ & 318.5 & 0.0 & $11,902.5$ & $4,323.0$ & 524.0 & 4066.3 & 334.4 & 0.0 \\
\hline & $12,570.2$ & $4,307.9$ & 822.7 & 0.0 & $12,570.2$ & $4,689.8$ & 990.0 & $4,451.5$ & 863.9 & 0.0 \\
\hline
\end{tabular}

Table 5. Inevitable work overload values due to the production mixes $(\varepsilon)$, the limitation of the static average saturation $\left(\eta_{m e d}^{\infty}=0.95\right)$ and the activation level of workers $\left(\alpha_{i, k}\right)$; and work overload given by $M 3 \cup 4 \_\eta$, $M 4 \cup 3 \_\eta, M 3 \cup 4 \_\dot{\alpha} I \_\eta$ and $M 4 \cup 3 \_\dot{\alpha} I \_\eta$ models, with the work pace functions $\left(\dot{\alpha_{t}}\right)$.

As can be noted, the inevitable work overload is considerably reduced when the work pace of operators increases at some period of workday. Specifically, if the activity factor is increased through a stepped function, which only represents an average increase of $3.33 \%$, the inevitable work overload is $4,307.9 \mathrm{~s}$ on average. Similarly, whether the average activation is equal to $5 \%$, the work overload is $822.7 \mathrm{~s}$. However when the activation corresponds to $6.66 \%$, the inevitable work overload is completely eliminated. 
On the other hand, the models the $M 3 \cup 4 \_\dot{\alpha} I_{-} \eta$ and $M 4 \cup 3 \_\dot{\alpha} I \_\eta$ also have reached all the optimal solutions, such as the case of the first computational experience. Nevertheless, now, these solutions do not coincide with the inevitable values. Indeed the $M 3 \cup 4 \_\dot{\alpha} I_{-} \eta$ and $M 4 \cup 3 \_\dot{\alpha} I_{-} \eta$ worsen the work overload value by 8.9 and $24.8 \%$, on average, when the stepped and the triangular functions are considered. Even so the incorporation of a largest activity factor at certain moments of the workday allows a reduction of the overall overload values. Specifically, the stepped function reduces the noncompleted work by $62.7 \%$, on average, in regard with the solutions given by $M 3 \cup 4 \_\eta$ and $M 4 \cup 3 \_\eta$ models; the triangular function decreases these values by $92.1 \%$ and both functions, the trapezoidal and parabolic, together with their corresponding functions with a mean linear activation are able to eliminate the $100 \%$ of the non-completed work.

\section{Result analysis}

Obviously, the activation of workers produces a productivity improvement even considering the saturation conditions established by the collective agreements. This improvement can be economically translated (€/year) whether the consolidated operation profit of the line (10\%), the total value of a engine (4000 Euros/engine), the production time per engine $(175 \mathrm{~s})$ and the working calendar (225 days/year) are taken into account.

However, these improvements are achieved by means of excess effort by workers, since they must increase their work pace at specific moments of their workday. Because of this and although demanded activity factor does not overtake the maximum value established by law $\left(\alpha_{i, k}^{*}=1.2\right)$ at no moment of the day, we calculate the extra cost resulting from the effort demanded to the workers. For that purpose and taking into account that our case study is located in Spain, we consider the current hourly cost per operator in the Spanish automotive sector (i.e. $[20 € / h ; 25 € / h]$ ). Therefore, according the used functions, the 21 workstations of the line and the two workers by processor, we will have the following costs:

- Stepped function: [81,860.63€/year - line; 102,235.78€/year - line].

- Triangular function: [124,031.25 €/year - line; 155,039.06€/year - line].

- Trapezoidal / Parabolic function: [165,358.46 €/year-line;206,718.54 €/ year - line].

Based on the above, we can calculate the net profits obtained by reduction of work overload by means of activation (Table 6). These profits are calculated considering, first, the number of uncompleted engines when saturation conditions are taken into account; second, the number of uncompleted engines when both saturation conditions and activation of workers are taken into account; and third, the rank of costs from the excess effort of workers according the functions for the work pace factor.

After confirming that the increase factor of work pace brings benefits when average dynamic saturation of processors is limited, including the compensation of operators for that excess effort, we analyze whether also the reinforcement of the line by auxiliary processors to reduce overload is feasible or not. 
Consideration of human resources in the Mixed-Model Sequencing Problem with Work Overload Minimization: Legal provisions and productivity improvement

J. Bautista, R. Alfaro-Pozo, C. Batalla-García

\begin{tabular}{cccccc}
\hline \multirow{2}{*}{$\begin{array}{c}\text { Net profit } \\
\text { M€/year-line })\end{array}$} & $\dot{\alpha}^{\mathrm{S}}$ & $\dot{\alpha}^{\mathrm{T}}$ & $\overline{5} \cup 4 \_\dot{\alpha} I_{-} \eta=M 4 \cup 3 \_\dot{\alpha} I_{-} \eta$ \\
\hline Min. & {$[3.81 ; 3.79]$} & {$[5.67 ; 5.64]$} & {$[3.94 ; 3.92]$} & {$[5.77 ; 5.74]$} & {$[5.90 ; 5.86]$} \\
Max. & {$[4.18 ; 4.16]$} & {$[5.89 ; 5.86]$} & {$[4.30 ; 4.28]$} & {$[5.91 ; 5.88]$} & {$[6.71 ; 6.67]$} \\
Average & {$[3.97 ; 3.95]$} & {$[5.83 ; 5.80]$} & {$[4.09 ; 4.07]$} & {$[5.90 ; 5.87]$} & {$[6.30 ; 6.26]$} \\
\hline
\end{tabular}

Table 6. Annual net profits (in millions of Euros) obtained by the reduction of work overload given by $M 3 \cup 4 \_\dot{\alpha} I_{-} \eta$ and $M 4 \cup 3 \_\dot{\alpha} I_{-} \eta$ models and taking into account the excess effort costs.

From the number of both multi-skilled and specialized auxiliary workers (equations 78 and 80) needed to eliminate the work overload in each studied scenario (Table 7), we can determine the cost of this approach addressed to counteract the negative effect of saturation limitations (Table 8). For this purpose we have taken into account the average number of auxiliary workers for both cases multi-skilled and specialized workers, the rank of the hourly cost per operator, and the cost of the activation of workers.

\begin{tabular}{|c|c|c|c|c|c|c|c|c|c|}
\hline \multirow{2}{*}{$\varepsilon$} & \multicolumn{3}{|c|}{$\Delta H\left(M 3 \cup 4 \_\dot{\alpha} I \_\eta=M 4 \cup 3 \_\dot{\alpha} I \_\eta\right)$} & \multicolumn{6}{|c|}{$\widehat{\Delta} H\left(M 3 \cup 4 \_\dot{\alpha} I \_\eta=M 4 \cup 3 \_\dot{\alpha} I \_\eta\right)$} \\
\hline & - & $\dot{\alpha}^{S}, \dot{\alpha}^{T}, \overline{\alpha^{S}}, \overline{\alpha^{T}}$ & $\dot{\alpha}^{Z}, \dot{\alpha}^{P}, \overline{\alpha^{Z}}, \overline{\alpha^{P}}$ & - & $\dot{\alpha}^{S}$ & $\dot{\alpha}^{T}$ & $\overline{\alpha^{s}}$ & $\overline{\alpha^{T}}$ & $\dot{\alpha}^{Z}, \dot{\alpha}^{P}$ \\
\hline$\# 1$ & 4 & 2 & 0 & 24 & 20 & 20 & 20 & 20 & 0 \\
\hline \#2 & 4 & 2 & 0 & 24 & 20 & 20 & 20 & 16 & 0 \\
\hline \#3 & 4 & 2 & 0 & 32 & 20 & 12 & 20 & 12 & 0 \\
\hline \#4 & 4 & 2 & 0 & 24 & 20 & 20 & 20 & 20 & 0 \\
\hline \#5 & 4 & 2 & 0 & 28 & 20 & 16 & 20 & 16 & 0 \\
\hline \#6 & 4 & 2 & 0 & 24 & 20 & 20 & 20 & 20 & 0 \\
\hline \#7 & 4 & 2 & 0 & 28 & 20 & 16 & 20 & 12 & 0 \\
\hline \#8 & 4 & 2 & 0 & 24 & 20 & 20 & 20 & 20 & 0 \\
\hline \#9 & 4 & 2 & 0 & 24 & 20 & 20 & 20 & 16 & 0 \\
\hline \#10 & 4 & 2 & 0 & 32 & 20 & 12 & 20 & 12 & 0 \\
\hline \#11 & 4 & 2 & 0 & 28 & 20 & 16 & 20 & 16 & 0 \\
\hline \#12 & 4 & 2 & 0 & 24 & 20 & 16 & 20 & 16 & 0 \\
\hline \#13 & 4 & 2 & 0 & 24 & 20 & 20 & 20 & 20 & 0 \\
\hline \#14 & 4 & 2 & 0 & 24 & 20 & 20 & 20 & 16 & 0 \\
\hline \#15 & 4 & 2 & 0 & 28 & 20 & 16 & 20 & 16 & 0 \\
\hline \#16 & 4 & 2 & 0 & 24 & 20 & 20 & 20 & 20 & 0 \\
\hline \#17 & 4 & 2 & 0 & 28 & 20 & 16 & 20 & 16 & 0 \\
\hline \#18 & 4 & 2 & 0 & 24 & 20 & 20 & 20 & 20 & 0 \\
\hline \#19 & 4 & 2 & 0 & 32 & 20 & 12 & 20 & 12 & 0 \\
\hline \#20 & 4 & 2 & 0 & 24 & 20 & 16 & 20 & 16 & 0 \\
\hline \#21 & 4 & 2 & 0 & 24 & 20 & 20 & 20 & 20 & 0 \\
\hline \#22 & 4 & 2 & 0 & 28 & 20 & 16 & 20 & 12 & 0 \\
\hline \#23 & 4 & 2 & 0 & 28 & 20 & 20 & 20 & 12 & 0 \\
\hline
\end{tabular}

Table 7. Number of multi-skilled auxiliary operators $(\Delta H)$ and specialized auxiliary operators $(\hat{\Delta} H)$ needed to eliminate the workload obtained with $M 3 \cup 4 \_\eta, M 4 \cup 3 \_\eta, M 3 \cup 4 \_\dot{\alpha} I_{-} \eta$ and $M 4 \cup 3 \_\dot{\alpha} I \_\eta$ models. 


\begin{tabular}{rrccccc}
\hline & M3 $\cup$ 4_d $\dot{\alpha} I_{-} \eta$ & \multicolumn{5}{c}{$M 3 \cup 4{ }_{-} \dot{\alpha} I_{-} \eta=M 4 \cup 3 \_\dot{\alpha} I_{-} \eta$} \\
\cline { 3 - 6 }$M 4 \cup 3 \_\dot{\alpha} I_{-} \eta$ & $\dot{\alpha}^{S}$ & $\dot{\alpha}^{T}$ & $\overline{\alpha^{S}}$ & $\overline{\alpha^{T}}$ & $\dot{\alpha}^{Z, P}$ \\
\hline$\Delta \mathrm{H}[115.6 ; 144.4]$ & {$[140.9 ; 176.1]$} & {$[183.1 ; 228.9]$} & {$[140.9 ; 176.1]$} & {$[183.1 ; 228.9]$} & {$[165.2 ; 206.5]$} \\
$\hat{\Delta} \mathrm{H}[775.5 ; 969.4]$ & {$[672.5 ; 840.6]$} & {$[714.7 ; 893.3]$} & {$[600.6 ; 750.6]$} & {$[606.8 ; 758.5]$} & {$[165.2 ; 206.5]$} \\
\hline
\end{tabular}

Table 8. Average costs (in thousands of Euros) from eliminating the work overload by means of hiring multiskilled $(\Delta H)$ and specialized $(\hat{\Delta} H)$ auxiliary workers and compensation of over-effort required to increase the work pace factor, considering the range of hourly cost per worker in Spain $[20 ; 25 € / h]$.

Obviously, it should be noted that the best situation, where the work overload is eliminated only by means of multi-skilled operators, may not be technically feasible because the workers must move along the line and one only worker cannot work at two stations simultaneously.

\section{Conclusions}

After establishing a set of legal bases on the working conditions in the automotive industry, such as the workday duration and the saturation of the workstations, we have proposed two equivalent mathematical models for the $M M S P-W$. These new models are focused on measuring the impact produced by such labor characteristics, on the work overload generated in a mixed-model assembly line.

The proposed models, $M 3 \cup 4 \_\eta$ and $M 4 \cup 3 \_\eta$, were applied to a case study of the Nissan engine plant in Barcelona that consist of 23 different demand plans. The computational experience determined that the improvement in working conditions designed to limit the average saturation of the workstations causes a drastic drop in production. This drop is estimated in terms of 75 daily engines in a line with a capacity of 270 engines in two shifts.

In order to relieve the production drop, we have proposed the activation of the processors and the incorporation of auxiliary processors at the assembly line.

The first proposal consists on increasing the work pace of operators (while always respecting the agreed working conditions) under the guidance of temporary functions of the activity factor that unfolds throughout the workday. This action has been concretized in two new models, the $M 3 \cup 4 \_\dot{\alpha} I \_\eta$ and $M 4 \cup 3 \_\dot{\alpha} I \_\eta$, which incorporate the activity factor in the execution times of the operations.

After running the models with saturation and activation conditions, we have observed how the economic losses derived from the number of uncompleted engines are reduced whenever the average level activity of workers increases. Indeed, from the losses due to the work overload given by $M 3 \cup 4 \_\eta$ and $M 4 \cup 3 \_\eta$ models, we have calculated the net profits that would be obtained by require workers more effort, considering the extra-cost that supposes the compensation of this over-effort at certain moments of workday. This has allowed us to observe, how whatever the increased activity of operators, benefits are obtained. 
However, the work pace rise does not clear the $100 \%$ of work overload for cases in which the increase is $3.33 \%$ or $5 \%$ (stepped and triangular function). For this reason and in order to assess the feasibility of incorporating auxiliary operators, we have also calculated the minimum and maximum reinforcement operators needed to achieve null work overload in all cases.

Thus, if we consider multi-skilled workers able to move about stations in a negligible time and able to develop any task, four workers will be needed to eliminate overloads obtained with the $M 3 \cup 4 \_\eta$ and $M 4 \cup 3 \_\eta$ models; 2 operators for overloads given by $M 3 \cup 4 \_\dot{\alpha} I_{-} \eta$ and $M 4 \cup 3 \_\dot{\alpha} I_{-} \eta$ models, when the activity factor follows a stepped or triangular function; and none to the other cases.

On the other hand, considering the extreme situation where the auxiliary workers are assigned to a single station because they are specialized, a maximum number of 32 operators are necessary when working at normal activity, 20 when the activity factor is increased by $3.33 \%$ (stepped function) and $5 \%$ (triangular function) on average and none when the average increase is $6.66 \%$.

Thus, given that the hourly cost per worker in the automotive sector in Spain ranges between 20 and $25 €$; the engine line represents a cost of $10 \%$ from value of a engine and therefore, a lost engine supposes a loss of $400 €$; and the work schedule is 225 working days; we have calculated the costs in regard with both the level activation of workers and the number of auxiliary processors (Fig. 5).

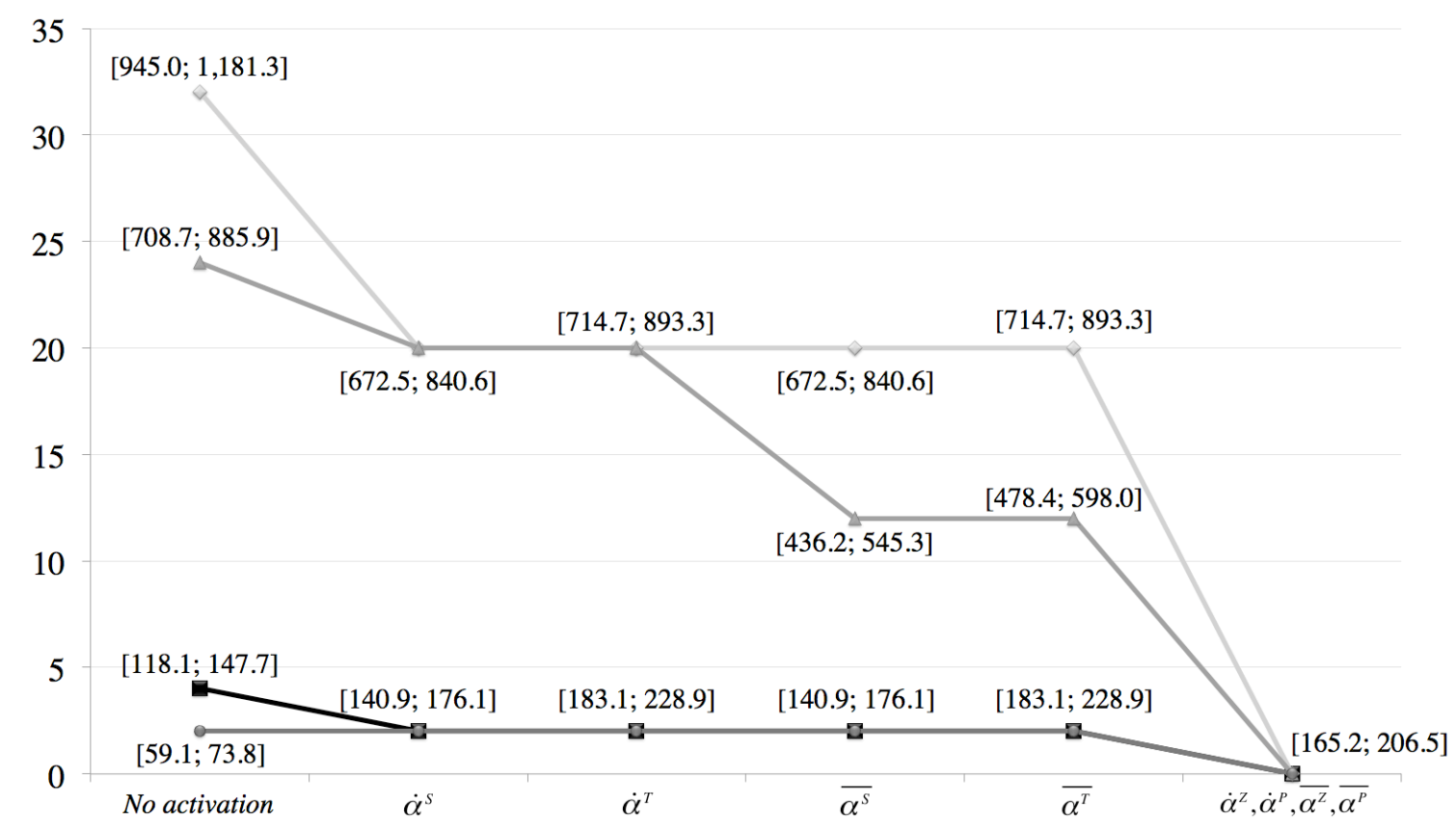

Fig 4. Maximum and minimum number of multi-skilled operators (black lines); the maximum and minimum specialized operators (gray lines) needed to completely eliminate the work overload associated to one workday composed by two shifts; and the cost range due to the reinforcement (considering that auxiliary operators work at normal activity) and the excess effort required to operators.

The results show that all evaluated scenarios reported profits because of none supposes a costs greater than the losses derived from the incorporation of saturation condition 
(6,464.6 thousands of Euros on average). Indeed, except in the case of trapezoidal or parabolic functions in which no auxiliary operators are required, net profits after combination of both measures (activation and reinforcement operators) are higher than those obtained with a single measure to reduce the effect of saturation and also to complete $100 \%$ of the required work.

It should be noted that the proposed models have been performed given a specific case study, however they models can be applied to other case studies whose characteristics are those of MMSP; only the parameters values of saturation and activation should be adapted to each case.

In short, the models proposed in this paper allow fulfilling the legal conditions imposed by collective agreements and, therefore, approach the problem from literature to real environments, all this without losing sight of the objective of completing all the required work and maximizing productivity. In other words, this study has simultaneously addressed merely productive aspects with social aspects that are increasingly important in industrial environments.

In future works, the incorporation of auxiliary processors will be studied more thoroughly. Specifically, an intermediate situation where there are specialized operators in the operations assigned to a set of consecutive workstations will be analyzed. Thus, a single processor may support a specific set of consecutive workstations overloaded, but not all at once, so that operators can move from one to another and do not require such demanding training degree. We also study an alternative to improve the average saturation by means of rotational programs among the workstations with the purpose of reducing the medium-and long-term saturation. Finally, in order to bring closer the $M M S P$-W to real and current situations of productive environments, we will incorporate, into the models proposed in this paper, JIT characteristics, with the purpose of improving the production regularity and thus favoring both a regular consumption of components and a regular use of resources.

\section{Acknowledgments}

The authors greatly appreciate the collaboration of Nissan Motor Ibérica (NMISA). This work was funded by projects PROTHIUS-III (DPI2010-16759) and FHI-SELM2 (TIN2014-57497-P) from the Spanish government.

\section{References}

Alfaro, R. (2015). Modelado y Resolución de Variantes del Problema de Secuenciación de Modelos Mixtos con Minimización de la Sobrecarga (MMSP-W), con Factores de Actividady Regularidad en la Producción. Ph.D. thesis. Universistat Politècnica de Catalunya, doi: 10.13140/2.1.1691.9362.

Battaïa, O., \& Dolgui, A. (2013). A taxonomy of line balancing problems and their solution approaches. International Journal of Production Economic, 142 (2), 259-277. 
Bautista, J., Alfaro, R., \& Batalla, C. (2015a). Modeling and solving the mixed-model sequencing problem to improve productivity. International Journal of Production Economic, $161,83-95$.

Bautista, J., Alfaro-Pozo, R., Batalla-Garcia, C., Llovera, S., et al. (2015b). MixedModel Sequencing Problem improving labour conditions. In P. Cortés, et al. (Eds.), Enhancing Synergies in a Collaborative Environment (1st ed., pp. 3-10). Springer International Publishing Lecture Notes in Management and Industrial Engineering Chapter 1, ISBN: 978-3-319-14077-3, doi: 10.1007/978-3-319-14078-0_1.

Bautista, J., Alfaro, R., Batalla, C., Cano, A., et al. (2014). Incorporating the Work Pace Concept into the MMSP-W. In C. Hernández, et al. (Eds), Managing complexity, challenges for industrial engineering and operations management(pp. 261-268). Springer International Publishing Lecture Notes in Management and Industrial Engineering ISBN: 978-3-319-04705-8, doi: 10.1007/978-3-319-04705-8_30.

Bautista, J., \& Cano, A. (2011). Solving mixed model sequencing problem in assembly lines with serial workstations with work overload minimisation and interruption rules. European Journal Operations Research, 210(3), 495-513., 210(3), 495-513.

Bautista, J., Cano, A., \& Alfaro, R. (2011). A bounded dynamic programming algorithm for the MMSP-W considering workstation dependencies and unrestricted interruption of the operations. In Proceedings of the 11th international conference on intelligent systems design and applications, ISDA2011ISBN: 978-145771675-1.

Bautista, J., Cano, A., \& Alfaro, R. (2012a). Modeling and solving a variant of the mixed-model sequencing problem with work overload minimisation and regularity constraints. An application in Nissan's Barcelona Plant. Expert System with Applications, 39(12), 11001-11010.

Bautista, J., Cano, A., \& Alfaro, R. (2012b). Models for MMSP-W considering workstation dependencies: A case study of Nissan's Barcelona Plant. European Journal Operations Research, 223(3), 669-679.

Bautista, J., Cano, A., Companys, R., \& Ribas, I. (2012). Solving the Fm|block|Cmax problem using Bounded Dynamic Programming. Engineering Applications of Artificial Intelligence, 25(6), 1235-1245.

Bautista, J., \& Suárez, R. (2009). Mixed-model sequencing problem with overload minimization considering workstations dependencies. In Proceedings of the IEEE international symposium on assembly and manufacturing, ISAM 2009 ISBN: 978142444627-8.

Becker, C., \& Scholl, A. (2006). A survey on problems and methods in generalized assembly line balancing. European Journal Operations Research, 168(3), 694-715. 
Boysen, N., Fliedner, M., \& Scholl, A. (2009a). Sequencing mixed-model assembly lines: Survey, classification and model critique. European Journal Operations Research, 192(2), 349-373.

Boysen, N., Fliedner, M., \& Scholl, A. (2009b).The product rate variation problem and its relevance in real world mixed-model assembly lines. European Journal Operations Research, 197(2), 818-824.

Celano, G., Costa, A., Fichera, S., \& Perrone, G. (2004). Human factor policy testing in the sequencing of manual mixed model assembly lines. Computer\&Operations Research, 31(1), 39-59.

Elmaghraby, S.E. (1978). Economic Lot Scheduling Problem (ELSP): Review and extensions. Management Science, 24(6), 587-598.

Giard, V., \& Jeunet, J. (2010). Optimal sequencing of mixed models with sequencedependent setups and utility workers on an assembly line. International Journal of Production Economic, 123(2), 290-300.

Llovera, S., Bautista, J., Llovera, J., \& Alfaro, R. (2014). Tiempo efectivo de trabajo: Un análisis normativo de la Jornada Laboral en el Sector de Automoción. Universistat Politècnica de Catalunya - BarcelonaTech, OPE-WP.2014/06 (http://hdl.handle.net/2117/24508), doi: $10.13140 / 2.1 .1946 .3680$.

Lin, D.-Y., \& Chu, Y.-M. (2013). The mixed-product assembly line sequencing problem of a door-lock company in Taiwan. Computer \& Industrial Engineering, 64(1), 492-499.

Lin, D.-Y., \& Chu, Y.-M. (2014). A Lagrangian relaxation approach to the mixedproduct assembly line sequencing problem: A case study of a door-lock company in Taiwan. Applied Mathematical Modelling, 38(17-18), 4493-4511.

Miltenburg, J. (1989). Level Schedules for Mixed-Model Assembly Lines in Just-InTime Production Systems. Management Science, 35(2), 192-207.

Monden,Y. (1983). Toyota production system: practical approach to production management. Norcross, Georgia: Industrial Engineering and Management Press.

Muse, L., Harris, S., \& Field, H. (2003). Has the Inverted-U Theory of Stress and Job Performance Had a Fair Test? Human Performance, 16(4), 349-364.

Pan, Q.-K., \& Ruíz, R. (2013). A comprehensive review and evaluation of permutation flowshop heuristics to minimize flowtime. Computer Operations Research, 40(1), 117-128.

Parrello, B.D., Kabat, W.C., \& Wos, L. (1986). Job-shop scheduling using automated reasoning: A case study of the car-sequencing problem. Journal of Automated Reasoning, 2(1), $1-42$. 
Raza, A.S., \& Akgunduz, A. (2008). A comparative study of heuristic algorithms on Economic Lot Scheduling Problem. Computer \& Industrial Engineering, 55(1), 94-109.

Salveson, M. E. (1955). The assembly line balancing problem. Journal of Industrial Engineering, 6(3), 18-25.

Scholl, A., Klein, R., \& Domschke, W. (1998). Pattern Based Vocabulary Building for Effectively Sequencing Mixed-Model Assembly Lines. Journal of Heuristics, 4(4), 359-381.

Solnon, C., Cung, V.D., Nguyen, A., \& Artigues, C. (2008). The car sequencing problem: Overview of state-of-the-art methods and industrial case-study of the ROADEF'2005 challenge problem. European Journal Operations Research, 191(3), 912927.

Yano, C.A., \& Rachamadugu, R. (1991). Sequencing to Minimize Work Overload in Assembly Lines with Product Options. Management Science, 37(5), 572-586. 